\title{
Multipolar Approach for Description of Bremsstrahlung During $\alpha$-Decay and Unified Formula of the Bremsstrahlung Probability
}

\author{
Sergei P. Maydanyuk ${ }^{*}$ \\ Institute for Nuclear Research, National Academy of Science of Ukraine, 47, prosp. Nauki, Kiev, 03680, Ukraine
}

\begin{abstract}
Improved multipolar model of photon bremsstrahlung accompanying $\alpha$-decay is presented. A special emphasis is given to the development of an angular formalism of matrix elements. The model gives values of the angular probability of the emission of photons in the absolute scale, without its normalization on experimental data. Spectra calculated on the basis of the model are found in a good agreement with the newest experimental data for the ${ }^{210} \mathrm{Po},{ }^{214} \mathrm{Po}$, and ${ }^{226} \mathrm{Ra}$ nuclei. A unified formula for the bremsstrahlung, probability during the $\alpha$-decay of an arbitrary nucleus, defined directly on $Q_{\alpha}$-value and numbers $A_{\mathrm{d}}, Z_{\mathrm{d}}$ of nucleons and protons of this nucleus, has been constructed for the first time. Inside the region of the $\alpha$-decaying nuclei from ${ }^{106} \mathrm{Te}$ up to nucleus with $A_{\mathrm{p}}=266$ and $Z_{\mathrm{p}}=109$ at energy of the photons emitted from $50 \mathrm{keV}$ up to $900 \mathrm{keV}$ a good coincidence has been achieved between the spectra obtained by the multipolar model (where duration of calculations for one selected nucleus is up to 1 day), and the spectra obtained by the unified formula (where duration of calculations is about some seconds, using the same computer).
\end{abstract}

Pacs: 23.60.+e, 41.60.-m, 23.20.Js, 03.65.Xp, 27.80.+w

Keywords: Bremsstrahlung, alpha-decay, formula for bremsstrahlung probability, tunneling.

\section{INTRODUCTION}

For the last two decades, many experimental and theoretical efforts have been made to investigate the nature of the bremsstrahlung emission accompanying $\alpha$-decay of heavy nuclei. A key idea of such researches consists of finding a new method of extraction of a new information about dynamics of $\alpha$-decay (and dynamics of tunneling) from measured bremsstrahlung spectra. The tunneling time in nuclear processes has extremely small values, close to nuclear one. This fact results almost in impossibility to test experimentally the non-stationary methods of the tunneling. But with the study, researchers open new ways for obtaining new information about dynamics of nuclear processes. An increasing interest in the study of the bremsstrahlung processes accompanying $\alpha$-decay is mainly explained by this, through analysis of the bremsstrahlung spectrum to estimate dynamics of the $\alpha$-decay (perhaps, in its first stage) or to estimate the duration of tunneling of the $\alpha$-particle through the nuclear barrier.

At present, there are many approaches for the description of the bremsstrahlung emission accompanying the $\alpha$-decay. In particular, a main emphasis has been given to such theoretical investigations where the $\alpha$-decay was considered as the semiclassical spherically symmetric approximation (for example, see Refs. [1-3]). The semiclassical approach in a comparison with fully quantum approach allows working with such characteristics and parameters, for which the

*Address correspondence to this author at the Institute for Nuclear Research, National Academy of Science of Ukraine, 47, prosp. Nauki, Kiev, 03680, Ukraine; E-mail: maidan@kinr.kiev.ua physical sense is obvious. This allows to understand new questions quicker in this task. At present, in the semiclassical approach an enough good description of existing experimental data has been achieved, where a recent success in agreement between theory and experiment for the controversial ${ }^{210}$ Po nucleus $[4,5]$ has been noted. Note good perspectives in the study of dynamics of the $\alpha$-decay with the analysis of the bremsstrahlung spectra [6-8], in the study of dynamics of tunneling in the $\alpha$-decay directly [9-12], and also an effect [13] named as Münchhausen effect, which increases the barrier penetrability due to virtual photon emission during its tunneling and which can be interesting for further study of the photon bremsstrahlung during tunneling in the $\alpha$-decay.

However, the fully developed quantum approach (for example, see Refs. [14-17]) is the most accurate and corrected description of emission of photons; the richest in the study of quantum properties and new effects in this task. In direction of development of the fully developed quantum approaches, a model proposed for the first time by Papenbrock and Bertsch in Ref. [14] has been developed, mainly where wave function of photons is considered in the dipole approximation. It turns out that application of such approach for calculation of the matrix element of the photon emission increases essentially its convergence without visible decreasing of accuracy, that makes this problem to be studied in the fully developed quantum approach practically for many researchers. An angular quantum model had been developed where further angular corrections in description of the wave function of photons are taken into account and potential of interactions between the $\alpha$-particle and the daughter nucleus is used in realistic form, according to Ref. [18]. Within the frameworks of this model, two different approaches have 
been developed for the calculation of the bremsstrahlung probability, based on different expansion of the wave function of photons: multipole expansion [19], and expansion in spherical waves $[20,21]$.

In the second approach, enough good agreement have been achieved with experimental data for the ${ }^{210} \mathrm{Po},{ }^{214} \mathrm{Po}$ and ${ }^{226} \mathrm{Ra}$ nuclei $[22,23]$. The multipolar approach is less developed. At the same time, it is more accurate in angular description of the photons emission during the $\alpha$-decay, and therefore, there is an interest in improved realization of this approach to the needed level. It turns out that the model constructed within the multipolar approach allows to calculate absolute values of the bremsstrahlung probability without any normalization relatively experimental data, and achieves good enough agreement with them. This opens a possibility to study the bremsstrahlung in the $\alpha$-decays of other nuclei and to predict new spectra. At present, it is unclear as to how much energy of the $\alpha$-particle takes influence on the bremsstrahlung emission, and whether other characteristics which influence the bremsstrahlung emission, are essential. On such a basis, more intriguing task has been opened to compose a unified formula of the bremsstrahlung probability during the $\alpha$-decay of the arbitrary nucleus, which is directly expressed through all these parameters and characteristics.

This paper answers on these questions, which are organized so. At first, the improved multipolar model of the bremsstrahlung accompanying the $\alpha$-decay is presented, where emphasis is made on construction of the angular formalism of the matrix elements and calculation of the absolute bremsstrahlung probability. Nucleus- $\alpha$-particle potential in the model, whose parameters are defined only by $\mathrm{Q}_{\alpha}$-value of the $\alpha$-decay, protons and neutron numbers for the studied nucleus, allows to apply this model for calculation of the absolute bremsstrahlung probability for arbitrary nucleus. Further, the model is tested in comparison of the calculated values of such probability with experimental data [4, 22, 23] for the ${ }^{210} \mathrm{Po},{ }^{214} \mathrm{Po}$ and ${ }^{226} \mathrm{Ra}$ nuclei and I have been obtaining good agreement. At the end, the formula of the bremsstrahlung probability during the $\alpha$-decay, based only on the $\mathrm{Q}_{\alpha}$-value and numbers of protons and neutrons of the decaying nucleus, has been constructed for the first time. Inside region of the $\alpha$-active nuclei from ${ }^{106} \mathrm{Te}$ up to the nucleus with numbers of nucleons and protons $A_{\mathrm{p}}=266$ and $Z_{\mathrm{p}}=109$ (this region is taken from Ref. [24]) with energy of the photons emitted from $50 \mathrm{keV}$ up to $900 \mathrm{keV}$, a satisfactory agreement has been achieved between the spectra, obtained on the basis of the multipolar model (where duration of calculations for one selected nucleus is up to 1 day), and the bremsstrahlung spectra obtained on the basis of the proposed formula (where duration of calculations is about some seconds using the same computer!). By the opinion of author, this formula can be extremely useful for the quick estimation of the bremsstrahlung probability during the $\alpha$ decay of the interesting nucleus (without a necessity to study enough complicated quantum models and variety of approximations, to realize enough laborious numerical algo- rithms of computer calculations of the bremsstrahlung spectra with resolution of divergence problem).

\section{MODEL}

\section{A. Matrix Element of Transition}

Let's formulate the starting points of the model. The bremsstrahlung probability has been defined during $\alpha$-decay of nucleus in terms of transition matrix element of the composite system ( $\alpha$-particle and daughter nucleus) from its state before the photon emission (we call such a state as initial $i$ state) into its state after the photon emission (we call such a state as final $f$-state). Index $i$ or $f$ to different characteristics and possible quantum numbers has been added for the initial or final state correspondingly, marking such state. The definition of the matrix element has been used like eq. (2.11) in Ref. [19] (in the first correction of the non-stationary perturbation theory with stationary limits $t_{0} \rightarrow-\infty$ and $t_{1} \rightarrow+\infty$, and with normalization $|C| \rightarrow 1$ ):

$a_{f i}=F_{f i} \cdot 2 \pi \delta\left(w_{f}-w_{i}+w_{\mathrm{ph}}\right)$,

where

$$
\begin{aligned}
F_{f i} & =Z_{e f f} \frac{e}{m} \sqrt{\frac{2 \pi \hbar}{w_{\mathrm{ph}}}} \cdot p\left(k_{i}, k_{f}\right), \\
p\left(k_{i}, k_{f}\right) & =\sum_{\alpha=1,2} \mathbf{e}^{(\alpha)^{*}} \cdot \mathbf{p}\left(k_{i}, k_{f}\right), \\
\mathbf{p}\left(k_{i}, k_{f}\right) & =\left\langle k_{f}\left|e^{-i \mathbf{k}_{\mathrm{ph}} \mathbf{r}} \frac{\partial}{\partial \mathbf{r}}\right| k_{i}\right\rangle= \\
& =\int \psi_{f}^{*}(\mathbf{r}) e^{-i \mathbf{k}_{\mathrm{ph}} \mathbf{r}} \frac{\partial \psi_{i}(\mathbf{r})}{\partial \mathbf{r}} \mathbf{d r},
\end{aligned}
$$

and $\psi_{i}(\mathbf{r})=\left|k_{i}\right\rangle$ and $\psi_{f}(\mathbf{r})=\left|k_{f}\right\rangle$ are stationary wave functions of the $\alpha$-decaying system in the initial $i$-state and final $f$-state, which do not contain number of photons emitted, $k_{i}=\sqrt{2 m E_{i}} / \hbar$ and $k_{f}=\sqrt{2 m E_{f}} / \hbar$ are wave vectors in the initial and final states. $E_{i}=\hbar w_{i}$ and $E_{f}=\hbar w_{f}$ are energies of the system in the initial and final states. $Z_{\text {eff }}=\left(2 A_{d}-4 Z_{d}\right) /\left(A_{d}+4\right)$ is an effective charge of the system, $m$ is reduced mass of this system, $A_{\mathrm{d}}$ and $Z_{\mathrm{d}}$ are numbers of nucleons and protons of the daughter nucleus. Further, an ndex ph has been added, marking different characteristics for the emitted photon. In particular, $\mathbf{k}_{p h}$ is a wave vector (momentum) of the emitted photon, $k_{\mathrm{ph}}$ is its modulus and $w_{p h}=k_{p h}=\left|\mathbf{k}_{p h}\right| \cdot \mathbf{e}^{(\alpha)}$ are unit vectors of polarization of this photon. Vectors $\mathbf{e}^{(\alpha)}$ are perpendicular to $\mathbf{k}_{p h}$ in Coulomb gauge. We have two independent polarizations $\mathbf{e}^{(1)}$ and $\mathbf{e}^{(2)}$ for the photon with momentum $\mathbf{k}_{p h}(\alpha=1,2)$. One can develop formalism simply in the system of units where $\hbar=1$ and $c=1$, but constants $\hbar$ and $c$ have been written explicitly. Let's find also square of the matrix element $a_{f i}$ used in definition of probability of transition. Using the formula of power reduction of $\delta$-function (see Ref. [25], § 21, p. 169): 


$$
\begin{aligned}
{[\delta(w)]^{2} } & =\delta(w) \delta(0)=\delta(w)(2 \pi)^{-1} \int d t= \\
& =\delta(w)(2 \pi)^{-1} T,
\end{aligned}
$$

we find ( $T \rightarrow+\infty$ is higher time limit):

$\left|a_{f i}\right|^{2}=2 \pi T\left|F_{f i}\right|^{2} \cdot \delta\left(w_{f}-w_{i}+w_{\mathrm{ph}}\right)$,

that looks like (4.21) in Ref. [25] (with accuracy up to factor $\left.(2 \pi)^{2}\right)$ and like (42.5) in Ref. [26] (exactly, see $\S 42$, p. 189).

\section{B. Linear and Circular Polarizations of the Photon Emit- ted}

Rewrite vectors of linear polarization $\mathbf{e}^{(\alpha)}$ through vectors of circular polarization $\xi_{\mu}$ with opposite directions of rotation (see Ref. [27], eq. (2.39), p. 42):

$\xi_{ \pm 1}=\mp \frac{1}{\sqrt{2}}\left(\mathbf{e}^{(1)} \pm i \mathbf{e}^{(2)}\right), \quad \xi_{0}=\mathbf{e}^{(3)}=0$.

Then $p\left(k_{i}, k_{f}\right)$ can be rewritten so:

$$
\begin{aligned}
& p\left(k_{i}, k_{f}\right)=\sum_{\mu= \pm 1} h_{\mu} \xi_{\mu}^{*} \int \psi_{f}^{*}(\mathbf{r}) e^{-i \mathbf{k}_{\mathrm{ph}} \mathbf{r}} \frac{\partial \psi_{i}(\mathbf{r})}{\partial \mathbf{r}} \mathbf{d r}, \\
& h_{ \pm}=\mp \frac{1 \pm i}{\sqrt{2}}, \quad h_{-1}+h_{+1}=-i \sqrt{2} \\
& \sum_{\alpha=1,2} \mathbf{e}^{(\alpha), *}=h_{-1} \xi_{-1}^{*}+h_{+1} \xi_{+1}^{*} .
\end{aligned}
$$

\section{Expansion of the Vector Potential A by Multipoles}

In further calculations of $p\left(k_{i}, k_{f}\right)$, the different expansions of function $e^{-i \mathbf{k}_{\mathrm{ph}} \mathbf{r}}$ of the vector potential $\mathbf{A}$ of the electro-magnetic field of the daughter nucleus can be used. In this paper, the multipole expansion has been used, defining it according to Ref. [27] (see eq. (2.106) in p. 58) so:

$$
\begin{aligned}
\xi_{\mu} e^{i \mathbf{k}_{p h} \mathbf{r}} & =\mu \sqrt{2 \pi} \sum_{l_{p h}=1, v}\left(2 l_{p h}+1\right)^{1 / 2} i^{l_{p h}} D_{v \mu}^{l_{p h}}(\phi, \theta, 0) \times \\
& \times\left[\mathbf{A}_{l_{p h} v}(\mathbf{r}, M)+i \mu \mathbf{A}_{l_{p h} v}(\mathbf{r}, E)\right],
\end{aligned}
$$

where (see Ref. [27], eq. (2.73) in p. 49, eq.(2.80) in p. 51)

$$
\begin{aligned}
& \mathbf{A}_{l_{\mathrm{ph}} v}(\mathbf{r}, M)=j_{l_{\mathrm{ph}}}\left(k_{\mathrm{ph}} r\right) \mathbf{T}_{l_{\mathrm{ph}} l_{\mathrm{ph}}, v}\left(\mathbf{n}_{\mathrm{ph}}\right), \\
& \mathbf{A}_{l_{\mathrm{ph}} v}(\mathbf{r}, E)=\sqrt{\frac{l_{\mathrm{ph}}+1}{2 l_{\mathrm{ph}}+1}} j_{l_{\mathrm{ph}}-1}\left(k_{\mathrm{ph}} r\right) \mathbf{T}_{l_{\mathrm{ph}} l_{\mathrm{ph}}-1, v}\left(\mathbf{n}_{\mathrm{ph}}\right)- \\
& -\sqrt{\frac{l_{\mathrm{ph}}}{2 l_{\mathrm{ph}}+1}} j_{l_{\mathrm{ph}}+1}\left(k_{\mathrm{ph}} r\right) \mathbf{T}_{l_{\mathrm{ph}} l_{\mathrm{ph}}+1, v}\left(\mathbf{n}_{\mathrm{ph}}\right) \text {. }
\end{aligned}
$$

Here, $\mathbf{A}_{l p h}(\mathbf{r}, M)$ and $\mathbf{A}_{l_{p h} v}(\mathbf{r}, E)$ are magnetic and electric multipoles, $j_{l}\left(k_{p h} r\right)$ are spherical Bessel functions of order $l_{p h}, \mathbf{T}_{l_{p h} l_{p h}, v}(\mathbf{n})$ are vector spherical harmonics, $\theta_{1}$, $\theta_{2}, \theta_{3}$ are angles defining direction of the vector $\mathbf{k}_{p h}$, relatively axis $z$, in selected frame system. According to Ref.
[27] (see comments in p. 51), in the case of $l_{p h}=0$, the multipoles $\mathbf{A}_{l_{p h} v}(\mathbf{r}, M)$ and $\mathbf{A}_{l_{p h} v}(\mathbf{r}, E)$ are zero. This corresponds to such physical fact that, photon with spin 1 must have at least unity of the angular momentum: $l_{p h}=1$. So, summation in eq. (8) was initiated from $l_{p h}=1$. Matrixfunction $D_{v \mu}^{l_{p h}}(\phi, \theta, 0)$ defines direction of the vector $\mathbf{k}_{p h}$ relatively axis $z$ in the frame system for $\mathbf{r}$ : angles $\phi$ and $\theta$ point to direction of the vector $\mathbf{k}_{p h}$, but not the vector $\mathbf{r}$. In general, the functions $\mathbf{T}_{j l, m}(\mathbf{n})$ have the following form $\left(\xi_{0}=0\right.$, see Ref. [27], p. 45):

$\mathbf{T}_{j l, m}(\mathbf{n})=\sum_{\mu= \pm 1}(l, 1, j \mid m-\mu, \mu, m) Y_{l, m-\mu}(\mathbf{n}) \xi_{\mu}$,

where $(l, 1, j \mid m-\mu, \mu, m)$ are Clebsh-Gordon coefficients (see Appendix A) and $Y_{l m}(\theta, \phi)$ are spherical functions defined according to Ref. [26] (see (B1) in Appendix B, also p. 119 , eqs. $(28,7)-(28,8))$.

\section{Approximation of the Spherically Symmetric $\alpha$-Decay}

Formula (8) is defined for a case when the vector $\mathbf{k}_{p h}$ is directed arbitrary, relatively arbitrary fixed system of coordinates. Let us orientate the system coordinates so that axis $z$ will be parallel to the vector $\mathbf{k}_{p h}$ and

$$
D_{\mu \nu}^{l_{p h}}(\varphi, \theta, 0)=\delta_{\mu \nu} \text {. }
$$

Then, eq. (8) is transformed into the following (see Ref. [27], eq. (2.105) in p. 57):

$$
\begin{aligned}
\xi_{\mu} e^{i \mathbf{k}_{p h} \mathbf{r}} & =\mu \sqrt{2 \pi} \sum_{l_{p h}=1}\left(2 l_{p h}+1\right)^{1 / 2} i^{l^{l p h}} \times \\
& \times\left[\mathbf{A}_{l_{p h} \mu}(\mathbf{r}, M)+i \mu \mathbf{A}_{l_{p h} \mu}(\mathbf{r}, E)\right] .
\end{aligned}
$$

This formula is convenient for a case when in a study of the $\alpha$-decay, the system of coordinates soes not needs to be fix by a definite way, relatively by the $\alpha$-decaying system (or the $\alpha$-decaying nucleus). This is a case when the $\alpha$-decaying system is considered in the spherically symmetric approximation. If the $\alpha$-decay has asymmetry then it needs to fix the system of coordinates, relatively the nucleus that decays. In such a case, the vector $\mathbf{k}_{p h}$ and axis $z$ cannot be parallel and they need to use eq. (8).

Further, the $\alpha$-decay in the spherically symmetrical approximation has been studied. In such a case, the wave functions of the $\alpha$-decaying system in the initial and final states are separated into the radial and angular components. Now these states are characterized by orbital and magnetic quantum numbers $l$ and $m$ and index $i$ or $f$ marking the initial or the final state has been added. It was assumed that in the initial state we have $l_{i}=m_{i}=0$ (like our previous papers [1923] and different other papers at this topic, see for example $[1-8,14-17])$. The interest was developed in such photon emission when the system transits to superposition of all possible final states with different values of the magnetic number $m_{f}$ at the same orbital number $l_{f}$. The radial compo- 
nent of the wave function $\varphi_{f}(r)$ does not depend on $m_{f}$ for any selected $l_{f}$. At present state, no restrictions have been applied for the possible values of $l_{f}$, i. e. it is assumed that $l_{f}$ can be arbitrary starting from 0 . Now, we write the wave functions so:

$\psi_{i}(\mathbf{r}) \quad=\varphi_{i}(r) Y_{00}\left(\mathbf{n}_{r}^{i}\right)$

$\psi_{f}\left(l_{f}, \mathbf{r}\right)=\varphi_{f}\left(l_{f}, r\right) \sum_{m_{f}} Y_{l_{f} m_{f}}\left(\mathbf{n}_{r}^{f}\right)$

and obtain:

$p\left(k_{i}, k_{f}\right)=\sqrt{2 \pi} \sum_{l_{p h}=1}(-1)^{l_{p h}} \sqrt{2 l_{p h}+1}\left(p_{l_{p h}}^{M}-i p_{l_{p h}}^{E}\right)$,

where

$p_{l_{p h}}^{M}=\sum_{\mu= \pm 1} \mu h_{\mu} p_{l_{p h} \mu}^{M}, \quad p_{l_{p h}}^{E}=\sum_{\mu= \pm 1} h_{\mu} p_{l_{p h}}^{E}$,

$p_{l_{p h} \mu}^{M}=\int_{0}^{+\infty} d r \int d \Omega r^{2} \psi_{f}^{*}\left(l_{f}, \mathbf{r}\right)\left(\frac{\partial}{\partial \mathbf{r}} \psi_{i}(\mathbf{r})\right) \mathbf{A}_{l_{p h} \mu}^{*}(\mathbf{r}, M)$,

$p_{l_{p h} \mu}^{E}=\int_{0}^{+\infty} d r \int d \Omega r^{2} \psi_{f}^{*}\left(l_{f}, \mathbf{r}\right)\left(\frac{\partial}{\partial \mathbf{r}} \psi_{i}(\mathbf{r})\right) \mathbf{A}_{l_{p h} \mu}^{*}(\mathbf{r}, E)$. [27]):

Using gradient formula (see eq. (2.56), p. 46 in Ref.

$$
\begin{aligned}
\frac{\partial}{\partial \mathbf{r}} f(r) Y_{l m}\left(\mathbf{n}_{r}\right) & =\sqrt{\frac{l}{2 l+1}}\left\{\frac{d f}{d r}+\frac{l+1}{r} f\right\} \mathbf{T}_{l l-1, m}\left(\mathbf{n}_{r}\right)- \\
& -\sqrt{\frac{l+1}{2 l+1}}\left\{\frac{d f}{d r}-\frac{l}{r} f\right\} \mathbf{T}_{l l+1, m}\left(\mathbf{n}_{r}\right)
\end{aligned}
$$

we obtain:

$\frac{\partial}{\partial \mathbf{r}} \psi_{i}(\mathbf{r})=-\frac{d \varphi_{i}(r)}{d r} \mathbf{T}_{01,0}\left(\mathbf{n}_{r}^{i}\right)$,

and then, we calculate the partial magnetic and electric components:

$$
\begin{aligned}
p_{l_{p h}}^{M} & =-I_{M}\left(l_{f}, l_{p h}, l_{p h}\right) \cdot J\left(l_{f}, l_{p h}\right), \\
p_{l_{p h}}^{E} & =-\sqrt{\frac{l_{p h}+1}{2 l_{p h}+1}} I_{E}\left(l_{f}, l_{p h}, l_{p h}-1\right) \cdot J\left(l_{f}, l_{p h}-1\right)+ \\
& +\sqrt{\frac{l_{p h}}{2 l_{p h}+1}} I_{E}\left(l_{f}, l_{p h}, l_{p h}+1\right) \cdot J\left(l_{f}, l_{p h}+1\right),
\end{aligned}
$$

where the following integral functions are introduced (at arbitrary $n=0,1,2 \ldots)$ :

$$
J\left(l_{f}, n\right)=\int_{0}^{+\infty} \varphi_{f}^{*}\left(l_{f}, r\right) \frac{d \varphi_{i}(r)}{d r} j_{n}\left(k_{p h} r\right) r^{2} d r,
$$

$$
\begin{aligned}
I_{M}\left(l_{f}, l_{p h}, n\right) & =\sum_{\mu= \pm 1} \mu h_{\mu} \cdot \int Y_{l_{f} m_{f}}^{*}\left(\mathbf{n}_{r}^{f}\right) \mathbf{T}_{01,0}\left(\mathbf{n}_{r}^{i}\right) \mathbf{T}_{l_{p h} n, \mu}^{*}\left(\mathbf{n}_{p h}\right) d \Omega, \\
I_{E}\left(l_{f}, l_{p h}, n\right) & =\sum_{\mu= \pm 1} h_{\mu} \cdot \int Y_{l_{f} m_{f}}^{*}\left(\mathbf{n}_{r}^{f}\right) \mathbf{T}_{01,0}\left(\mathbf{n}_{r}^{i}\right) \mathbf{T}_{l_{p h} n, \mu}^{*}\left(\mathbf{n}_{p h}\right) d \Omega .
\end{aligned}
$$

Using the following value of the Clebsh-Gordon coefficient (see Appendix A):

$$
(110 \mid 1,-1,0)=(110 \mid-1,1,0)=\sqrt{\frac{1}{3}}
$$

from eqs. (10) and (18) we obtain:

$$
\begin{aligned}
\mathbf{T}_{01,0}\left(\mathbf{n}_{r}^{i}\right) & =\sum_{\mu= \pm 1}(110 \mid-\mu \mu 0) Y_{1,-\mu}\left(\mathbf{n}_{r}^{i}\right) \xi_{\mu}= \\
& =\sqrt{\frac{1}{3}} \sum_{\mu= \pm 1} Y_{1,-\mu}\left(\mathbf{n}_{r}^{i}\right) \xi_{\mu}, \\
\frac{\partial}{\partial \mathbf{r}} \psi_{i}(\mathbf{r}) & =-\sqrt{\frac{1}{3}} \frac{d \varphi_{i}(r)}{d r} \sum_{\mu= \pm 1} Y_{1,-\mu}\left(\mathbf{n}_{r}^{i}\right) \xi_{\mu}
\end{aligned}
$$

and for the angular integrals for transition into the superposition of all possible final $f$-states with different $m_{f}$ at the same $l_{f}$ from eq. (20) we obtain:

$$
\begin{aligned}
I_{M}\left(l_{f}, l_{p h}, n\right) & =\sqrt{\frac{1}{3}} \sum_{\mu= \pm 1} \mu h_{\mu} \sum_{\mu^{\prime}= \pm 1}\left(n, 1, l_{p h} \mid \mu-\mu^{\prime}, \mu^{\prime}, \mu\right) \times \\
& \times \int Y_{l_{f} m_{f}}^{*}\left(\mathbf{n}_{r}^{f}\right) Y_{1,-\mu^{\prime}}\left(\mathbf{n}_{\mathbf{r}}^{i}\right) Y_{n, \mu-\mu^{\prime}}^{*}\left(\mathbf{n}_{p h}\right) d \Omega, \\
I_{E}\left(l_{f}, l_{p h}, n\right) & =\sqrt{\frac{1}{3}} \sum_{\mu= \pm 1} h_{\mu} \sum_{\mu^{\prime}= \pm 1}\left(n, 1, l_{p h} \mid \mu-\mu^{\prime}, \mu^{\prime}, \mu\right) \times \\
& \times \int Y_{l_{f} m_{f}}\left(\mathbf{n}_{r}^{f}\right) Y_{1,-\mu^{\prime}}\left(\mathbf{n}_{\mathbf{r}}^{i}\right) Y_{n, \mu-\mu^{\prime}}^{*}\left(\mathbf{n}_{p h}\right) d \Omega .
\end{aligned}
$$

\section{E. Vectors $\mathbf{n}_{r}^{i}, \mathbf{n}_{r}^{f}, \mathbf{n}_{p h}$ and Calculations of the Angular Integrals}

Let us analyze a physical sense of vectors $\mathbf{n}_{r}^{i}, \mathbf{n}_{r}^{f}$ and $\mathbf{n}_{p h}$. According to the definition of wave functions $\psi_{i}(\mathbf{r})$ and $\psi_{f}(\mathbf{r})$, the vectors $\mathbf{n}_{r}^{i}$ and $\mathbf{n}_{r}^{f}$ determine orientation of radius-vector $\mathbf{r}$ from the center of frame system to point where these wave functions describes the particle before and after the emission of photon. Such description of the particle has a probabilistic sense and is fulfilled over whole space. Change of direction of motion (or tunneling) of the particle in the result of the photon emission can be characterized by the change of quantum numbers $l$ and $m$ in the angular wave function: $Y_{00}\left(\mathbf{n}_{r}^{i}\right) \rightarrow Y_{l_{f} m_{f}}\left(\mathbf{n}_{r}^{f}\right)$ (which changes the probability of appearance of this particle along different directions, and angular asymmetry is appeared). The vector $\mathbf{n}_{p h}$ determines orientation of radius-vector $\mathbf{r}$ from the center of the frame system to point where the wave function of photon describes its "appearance". Using such logic, we have:

$\mathbf{n}_{p h}=\mathbf{n}_{r}^{i}=\mathbf{n}_{r}^{f}=\mathbf{n}_{r}$. 
As such a frame system has been used where axis $z$ is parallel to vector $\mathbf{k}_{p h}$ of the photon emission, then dependent on $\mathbf{r}$, integrant function in the matrix element represents amplitude (its square is probability) of appearance of the particle at point $\mathbf{r}$ after emission of photon, if this photon has emitted along axis $z$. Then, angle $\theta$ (of vector $\mathbf{n}_{\mathrm{r}}$ ) is the angle between direction of the particle motion (with possible tunneling) and direction of the photon emission.

Let us consider the angular integral in eq. (16) over $d \Omega$. Using eq. (17), we find:

$\int Y_{l_{f} m_{f}}^{*}\left(\mathbf{n}_{r}\right) Y_{1,-\mu^{\prime}}\left(\mathbf{n}_{r}\right) Y_{n, \mu-\mu^{\prime}}^{*}\left(\mathbf{n}_{\mathbf{r}}\right) d \Omega=(-1)^{l_{f}+n-\mu^{\prime}+1+\frac{\left|m_{f}+\mu^{\prime}\right|}{2}} i^{l_{f}+n+1} \times$

$\times \sqrt{\frac{3\left(2 l_{f}+1\right)(2 n+1)}{32 \pi} \frac{\left(l_{f}-1\right) !}{\left(l_{f}+1\right) !} \frac{\left(n-\left|m_{f}+\mu^{\prime}\right|\right) !}{\left(n+\left|m_{f}+\mu^{\prime}\right|\right) !}} \times$

$\times \int P_{l_{f}}^{1}(\cos \theta) P_{1}^{1}(\cos \theta) P_{n}^{\mid m_{f}+\mu^{\prime \mid}}(\cos \theta) \cdot \sin \theta d \theta d \phi$,

where $P_{l}^{m}(\cos \theta)$ are associated Legandre's polynomial (see Ref. [26], p. 752-754, eqs. (c,1)-(c,4); also see Ref. [27] eq. (2.6), p. 34), and we obtain the following restrictions on possible values of $m_{f}$ and $l_{f}$ (for more detais, see Appendix B):

$m_{f}=-\mu= \pm 1, \quad l_{f} \geq 1, \quad n \geq\left|\mu-\mu^{\prime}\right|=\left|m_{f}+\mu^{\prime}\right|$

when this integral is non-zero (in particular, at $l_{f}=0$ the integral in the left part of eq. (25) is zero).

Now let us introduce the following coefficient $C_{l_{f} l_{p h} m^{n}}^{m^{\prime}}$ :

$$
\begin{aligned}
C_{l_{f} l_{p h} n}^{m_{f} \mu^{\prime}} & =(-1)^{l_{f}+n+1-\mu^{\prime}+\frac{\left|m_{f}+\mu^{\prime}\right|}{2}}\left(n, 1, l_{p h} \mid-m_{f}-\mu^{\prime}, \mu^{\prime},-m_{f}\right) \times \\
& \times \sqrt{\frac{\left(2 l_{f}+1\right)(2 n+1)}{32 \pi} \frac{\left(l_{f}-1\right) !}{\left(l_{f}+1\right) !} \frac{\left(n-\left|m_{f}+\mu^{\prime}\right|\right) !}{\left(n+\left|m_{f}+\mu^{\prime}\right|\right) !}}
\end{aligned}
$$

and function $f_{l_{f} n}^{m_{f} \mu^{\prime}}(\theta)$ :

$f_{l_{f} n}^{m_{f} \mu^{\prime}}(\theta)=P_{l_{f}}^{1}(\cos \theta) P_{1}^{1}(\cos \theta) P_{n}^{\left|m_{f}+\mu^{\prime}\right|}(\cos \theta)$.

Then, after integrating over $\phi$, we find the total angular integrals $I_{M}\left(l_{f}, l_{p h}, n\right)$ and $I_{E}\left(l_{f}, l_{p h}, n\right)$ :

$I_{M}\left(l_{f} ; l_{p h}, n\right)=-m_{f} h_{-m} i^{l_{f}+n+1} \sum_{\mu^{\prime}= \pm 1} C_{l_{f} l_{p h} m^{m_{f}} \mu^{\prime}}^{\pi} \int_{0}^{\pi} f_{l_{f}{ }^{m_{f}} \mu^{\prime}}(\theta) \sin \theta d \theta$,

$I_{E}\left(l_{f} ; l_{p h}, n\right)=h_{-m} i^{l_{f}+n+1} \sum_{\mu^{\prime}= \pm 1} C_{l_{f} l_{p h} m^{m_{1}} \mu^{\prime}}^{\pi} \int_{0}^{\pi} f_{l_{f} n}^{m_{f} \mu^{\prime}}(\theta) \sin \theta d \theta$

Now, let us introduce the following differential expressions of these integrals by angle $\theta$ (neglecting by limits of integration):

$$
\begin{aligned}
& \frac{d I_{M}\left(l_{f} ; l_{p h}, n\right)}{\sin \theta d \theta}=-m_{p h} h_{-m} i^{l_{f}+n+1} \sum_{\mu^{\prime}= \pm 1} C_{l_{f} l_{p h} n}^{m_{f} \mu^{\prime}} f_{l_{f} n}^{m_{f} \mu^{\prime}}(\theta), \\
& \frac{d I_{E}\left(l_{f} ; l_{p h}, n\right)}{\sin \theta d \theta}=h_{-m} i^{l_{f}+n+1} \sum_{\mu^{\prime}= \pm 1} C_{l_{f} l_{p h} m^{\prime}}^{m_{1}} f_{l_{f} n^{\prime}}^{m_{f} \mu^{\prime}}(\theta) .
\end{aligned}
$$

On such a basis, new differential partial magnetic and electric components, $d p_{l_{p h}}^{M}$ and $d p_{l_{p h}}^{E}$, dependent on angle $\theta$ have been defined as:

$$
\begin{aligned}
& \frac{d p_{l_{p h}}^{M}}{\sin \theta d \theta}=-\frac{d I_{M}\left(l_{f}, l_{p h}, l_{p h}\right)}{\sin \theta d \theta} \cdot J\left(l_{f}, l_{p h}\right)= \\
& =m h_{-m} i^{l_{f}+l_{p h}+1} J\left(l_{f}, l_{p h}\right) \sum_{\mu^{\prime}= \pm 1} C_{l_{f} l_{p h} l_{p h} m^{\prime} \mu^{\prime}}^{m_{l_{f}} l_{p h}} f_{\mu^{\prime}}^{\mu^{\prime}}(\theta), \\
& \frac{d p_{l_{p h}}^{E}}{\sin \theta d \theta}=-h_{-m} i^{l_{f}+l_{p h}} \sqrt{\frac{l_{p h}+1}{2 l_{p h}+1}} J\left(l_{f}, l_{p h}-1\right) \sum_{\mu^{\prime}= \pm 1} C_{l_{f}, l_{p h}, l_{p h} m^{\prime} \mu^{\prime}} f_{l_{f}, l_{p h}-1}^{m_{f} \mu^{\prime}}(\theta)+ \\
& +h_{-m} l^{l_{f}+l_{p h}+2} \sqrt{\frac{l_{p h}}{2 l_{p h}+1}} J\left(l_{f}, l_{p h}+1\right) \sum_{\mu^{\prime}= \pm 1} C_{l_{f}, l_{p h}, l_{p h}+1}^{m_{p h}} f_{l_{f}, l_{p h}+1}^{m_{f} \mu^{\prime}}(\theta)
\end{aligned}
$$

and new total differential component $d p$

$$
\frac{d p\left(k_{i}, k_{f}\right)}{\sin \theta d \theta}=\sqrt{2 \pi} \sum_{l_{p h}=1}(-i)^{l_{p h}} \sqrt{2 l_{p h}+1} \cdot\left(\frac{d p_{l_{p h}}^{M}}{\sin \theta d \theta}-i \frac{d p_{l_{p h}}^{E}}{\sin \theta d \theta}\right)
$$

dependent on this angle also. One can see that integration of the differential components (31) over the angle $\theta$ with limits from 0 to $\pi$ gives the integral components $p_{l_{p h}}^{M}$ and $p_{l_{p h}}^{E}$ exactly.

For transition into superposition of all possible final states with different $m_{f}$ at the same $l_{f}$ instead of eq. (31), we obtain:

$$
\begin{aligned}
& \frac{d \tilde{p}_{l_{p h}}^{M}}{\sin \theta d \theta}=i^{l_{f}+l_{p h}+1} J\left(l_{f}, l_{p h}\right) \sum_{m_{f}= \pm 1} m_{f} h_{-m} \sum_{\mu^{\prime}= \pm 1} C_{l_{f}^{\prime} p h h^{\prime} l_{l}}^{m_{j} \mu^{\prime}} f_{l_{f} l_{p h}}^{m_{f} \mu^{\prime}}(\theta), \\
& \frac{d \tilde{p}_{l_{p h}}^{E}}{\sin \theta d \theta}=-i^{l_{f}+l_{p h}} \sqrt{\frac{l_{p h}+1}{2 l_{p h}+1}} J\left(l_{f}, l_{p h}-1\right) \sum_{m_{f}= \pm 1} h_{-m_{f}} \sum_{\mu^{\prime}= \pm 1} C_{l_{f}, l_{p h}, l_{p h}-1}^{m_{f} \mu^{\prime}} f_{l_{f}, l_{p h}-1}^{m_{j} \mu^{\prime}}(\theta)- \\
& \text { - } i^{l_{f}+l_{p h}} \sqrt{\frac{l_{p h}}{2 l_{p h}+1}} J\left(l_{f}, l_{p h}+1\right) \sum_{m_{f}= \pm 1} h_{-m_{f}} \sum_{\mu^{\prime}= \pm 1} C_{l_{f}, l_{p h} l_{p h}+l^{\prime}+1}^{\mu^{\prime}} f_{l_{f}, l_{p h}+1}^{m_{\mu} \mu^{\prime}}(\theta) \text {. }
\end{aligned}
$$

\section{F. The Components $p_{l_{p h}}^{M}$ and $p_{l_{p h}}^{E}$ at the First Values of $l_{f}, l_{p h}$}

The differential components (33) have been defined at the first values of $l_{f}$ and $l_{p h}$. Following eqs. (14) and (26), we have:

$l_{f}=1, \quad l_{p h}=1$

and we write:

$$
\begin{aligned}
\frac{d \tilde{p}_{1}^{M}}{\sin \theta d \theta} & =-i J(1,1) \cdot \sum_{m_{f}= \pm 1} m_{f} h_{-m} \sum_{\mu^{\prime}= \pm 1} C_{111}^{m_{f} \mu^{\prime}} f_{11}^{m_{f} \mu^{\prime}}(\theta), \\
\frac{d \tilde{p}_{1}^{E}}{\sin \theta d \theta} & =\sqrt{\frac{2}{3}} J(1,0) \sum_{m_{f}= \pm 1} h_{-m_{f}} \sum_{\mu^{\prime}= \pm 1} C_{110}^{m_{f} \mu^{\prime}} f_{10}^{m_{f} \mu^{\prime}}(\theta)+ \\
& +\sqrt{\frac{1}{3}} J(1,2) \sum_{m= \pm 1} h_{-m_{f}} \sum_{\mu^{\prime}= \pm 1} C_{112}^{m_{f} \mu^{\prime}} f_{12}^{m_{f} \mu^{\prime}}(\theta) .
\end{aligned}
$$

Calculating coefficients $C_{11 n}^{m_{f} \mu^{\prime}}$ and functions $f_{1 n}^{m_{f} \mu^{\prime}}(\theta)$ (see Appendixes C and D), we obtain: 


$$
\begin{aligned}
\frac{d \tilde{p}_{1}^{M}}{\sin \theta d \theta} & =-\frac{3}{8} \sqrt{\frac{1}{\pi}} \cdot J(1,1) \cdot \sin ^{2} \theta \cos \theta, \\
\frac{d \tilde{p}_{1}^{E}}{\sin \theta d \theta} & =i \frac{1}{8} \sqrt{\frac{2}{\pi}} \cdot J(1,0) \cdot \sin ^{2} \theta+ \\
& +i \frac{1}{8} \sqrt{\frac{1}{\pi}} \cdot J(1,2) \cdot \sin ^{2} \theta\left(1-3 \sin ^{2} \theta\right) .
\end{aligned}
$$

Integrating eqs. (36) over the angle $\theta$, we find the integral components:

$$
\tilde{p}_{1}^{M}=0, \quad \tilde{p}_{1}^{E}=i \frac{1}{6} \sqrt{\frac{2}{\pi}} \cdot\left(J(1,0)-\frac{7}{10} \sqrt{2} \cdot J(1,2)\right) .
$$

\section{G. Angular Probability of Emission of Photon with Momentum $k_{p h}$ and Polarization $\mathrm{e}^{(\alpha)}$}

The probability of transition of the system (during time unit) has been defined from the initial $i$-state into the final $f$ states, being in the given interval $d v_{f}$, with emission of photon with possible momenta inside the given interval $d v_{p h}$, so (see Ref. [26], $(42,5) \S 42$, p. 189; Ref. [28], § 44, p. 191):

$$
\begin{aligned}
& d W=\frac{\left|a_{f i}\right|^{2}}{T} \cdot d v=2 \pi\left|F_{f i}\right|^{2} \delta\left(w_{f}-w_{i}+w_{p h}\right) \cdot d v, \\
& d v=d v_{f} \cdot d v_{p h},
\end{aligned}
$$

where $d v$ are values characterizing photon and particle in the final $f$-state. If the emission of photon with momentum $\mathbf{k}_{p h}$ is considered then

$$
d v_{p h}=\frac{d^{3} k_{p h}}{(2 \pi)^{3}}=\frac{w_{p h}^{2} d w_{p h} d \Omega_{p h}}{(2 \pi c)^{3}}
$$

where $\quad d \Omega_{p h}=d \cos \theta_{p h}=\sin \theta_{p h} d \theta_{p h} d \phi_{p h}, \quad k_{p h}=w_{p h} / c$. Substituting eq. (39) into eq. (38) and integrating eq. (38) over $d w_{p h}$, we obtain:

$$
\begin{aligned}
& d W=\frac{w_{f i}^{2}\left|F_{f i}\right|^{2}}{(2 \pi)^{2} c^{3}} d \Omega_{p h} d v_{f}, \\
& w_{f i}=w_{i}-w_{f}=\frac{E_{i}-E_{f}}{\hbar} .
\end{aligned}
$$

Now, concerning interval $d v_{f}$ has been noted. In definition (38), we use matrix element $F_{f i}$, which is defined as an integral over space with possible summation by some quantum numbers of the system in the final $f$-state. One can consider such procedure as averaging by these characteristics, and then $F_{f i}$ does not depend on them. Therefore, we shall suppose that interval $d v_{f}$ in definition (38) takes into account only such additional characteristics and quantum numbers of the system in the final $f$-state, by which integration or summation was not fulfilled in definition of $F_{f i}$.
Substituting eq. (2) for $F_{f i}$ into eq. (40), we obtain:

$$
\begin{aligned}
d W & =\frac{Z_{\text {eff }}{ }^{2} e^{2}}{m^{2}} \frac{\hbar w_{f i}}{2 \pi c^{3}}\left|p\left(k_{i}, k_{f}\right)\right|^{2} d \Omega_{p h} d v_{f}= \\
& =\frac{Z_{\text {eff }}{ }^{2} \hbar e^{2}}{2 \pi c^{3}} \frac{w_{f i}}{m^{2}}\left|\sum_{\alpha=1,2} \mathbf{e}^{(\alpha), *} \mathbf{p}\left(k_{i}, k_{f}\right)\right|^{2} d \Omega_{p h} d v_{f} .
\end{aligned}
$$

This expression represents probability of the photon emission with momentum $\mathbf{k}_{p h}$ (and with averaging by polarization $\left.\mathbf{e}^{(\alpha)}\right)$ where the integration over angles of the particle motion after the photon emission has already fulfilled. Such probability is averaged over all possible directions of the particle motion after emission, and therefore, does not depend on them.

I define the following probability of emission of photon with momentum $\mathbf{k}_{p h}$ when after such emission the particle moves (or tunnels) along direction $\mathbf{n}_{\mathbf{r}}^{f}$ : differential probability concerning angle $\theta$ (and differential probability concerning solid angle $\Omega$ ) is such a function, definite integral of which over the angle $\theta$ with limits from 0 to $\pi$ (definite solid integral over angles $\theta$ and $\phi$ ) equals to the total probability of the photon emission (40). Let us consider two functions:

$$
\begin{aligned}
& \frac{d W\left(\phi_{f}, \theta_{f}\right)}{d \Omega_{p h} d \Omega_{f}}=\frac{d W\left(\phi_{f}, \theta_{f}\right)}{d \Omega_{p h} \sin \theta_{f} d \theta_{f} d \phi_{f}}=\frac{Z_{\text {eff }}{ }^{2} \hbar e^{2}}{2 \pi c^{3}} \frac{w_{f i}}{m^{2}} \frac{d}{d \Omega_{f}}\left|p\left(k_{i}, k_{f}\right)\right|^{2}, \\
& \frac{d W\left(\theta_{f}\right)}{d \Omega_{p h} d \cos \theta_{f}}=\frac{d W\left(\theta_{f}\right)}{d \Omega_{p h} \sin \theta_{f} d \theta_{f}}=\frac{Z_{\text {eff }}{ }^{2} \hbar e^{2}}{2 \pi c^{3}} \frac{w_{f i}}{m^{2}} \frac{d}{\sin \theta_{f} d \theta_{f}}\left|p\left(k_{i}, k_{f}\right)\right|^{2} .
\end{aligned}
$$

Using the definition (32) for the differential component $d p$, I rewrite eqs. (42) as:

$$
\begin{aligned}
& \frac{d W\left(\phi_{f}, \theta_{f}\right)}{d \Omega_{p h} d \Omega_{f}}=\frac{Z_{\text {eff }}{ }^{2} \hbar e^{2}}{2 \pi c^{3}} \frac{w_{f i}}{m^{2}}\left\{p\left(k_{i}, k_{f}\right) \frac{d p^{*}\left(k_{i}, k_{f}, \Omega_{f}\right)}{d \Omega_{f}}+\text { h.e. }\right\}, \\
& \frac{d W\left(\theta_{f}\right)}{d \Omega_{p h} d \cos \theta_{f}}=\frac{Z_{\text {eff }}{ }^{2} \hbar e^{2}}{2 \pi c^{3}} \frac{w_{f i}}{m^{2}}\left\{p\left(k_{i}, k_{f}\right) \frac{d p^{*}\left(k_{i}, k_{f}, \theta_{f}\right)}{d \cos \theta_{f}}+\text { h.e. }\right\} .
\end{aligned}
$$

One can see that so constructed functions satisfy exactly with the definition of the differential probabilities above. So, they can be used as definitions of the differential probabilities.

The total (integrated over angles) probability of the photon emission is:

$$
W=\frac{Z_{\mathrm{eff}}{ }^{2} \hbar e^{2}}{2 \pi c^{3}} \frac{w_{f i}}{m^{2}}\left|p\left(k_{i}, k_{f}\right)\right|^{2} .
$$

From eqs. (43) and (44) one can see that so defined angular probabilities are real.

If the probability $W$ has dimension of mass and coincides with width $\Gamma$ then one can define inverse value $\tau$ to it:

$$
\tau=\frac{\hbar}{\Gamma}, \quad \Gamma=W
$$


In consideration of transition of the system from the initial state to the final one, the value $\tau$ represents mean life time of this system in the initial state, i. e. before photon emission (see Ref. [25], p. 175).

The probability of photon emission is inversely proportional to the normalized volume $V$, which can be used arbitrary. With a purpose to obtain the characteristics, which characterizes the process of emission and does not depend on $V$, it needs to divide the differential probability of emission $d W$ on the flux $j$ of outgoing $\alpha$-particles in $\alpha$-decay, which is also inversely proportional to this volume $V$. Write the differential probability so:

$d W\left(\phi_{f}, \theta_{f}\right)=n_{i} v\left(\mathbf{p}_{i}\right) \cdot d P$,

where $n_{i}$ is the average number of particles in time unit before photon emission (used for normalization of wave function in the initial $i$-state, we have $\left.n_{i}=1\right), v\left(\mathbf{p}_{i}\right)$ is a module of velocity of outgoing particle in the system of coordinates where colliding center does not move (which coincides with laboratory frame where the second particle does not move). Factor $P$ is proportional to the element of the angle of the particle after its scattering as a result of photon emission and we shall call it as differential absolute probability (while value $d W$ we shall call as the relative probability).

Velocity of the particle with finite mass is (for example, see $\S 21.4$, p. 174 in Ref. [25] at $c=1$ ):

$\mathbf{v}=\frac{c^{2} \mathbf{p}}{E}, \quad v=|\mathbf{v}|=\frac{c^{2} p}{E}$.

Taking into account, that the wave functions of the particle before and after the emission have not momentums $\mathbf{p}_{i}$ and $\mathbf{p}_{f}$ of this particle, but they have the wave vectors $k_{i}$ and $k_{f}$, we rewrite eq. (47) so:

$v_{i}=\frac{\hbar c^{2} k_{i}}{E_{i}}$.

From here, we obtain equation of connection between differential relative and absolute probabilities:

$d P\left(\phi_{f}, \theta_{f}\right)=\frac{d W\left(\phi_{f}, \theta_{f}\right)}{n_{i} v(\mathbf{k})}=d W\left(\phi_{f}, \theta_{f}\right) \cdot \frac{E_{i}}{\hbar c^{2} k_{i}}$,

and find the final expression for the differential absolute probability $\left(n_{i}=1\right)$ :

$$
\begin{aligned}
& \frac{d P\left(\phi_{f}, \theta_{f}\right)}{d \Omega_{p h} d \Omega_{f}}=\frac{Z_{\text {eff }}{ }^{2} e^{2}}{2 \pi c^{5}} \frac{w_{p h} E_{i}}{m^{2} k_{i}}\left\{p\left(k_{i}, k_{f}\right) \frac{d p^{*}\left(k_{i}, k_{f}, \Omega_{f}\right)}{d \Omega_{f}}+\text { h.e. }\right\}, \\
& \frac{d P\left(\phi_{f}, \theta_{f}\right)}{d \Omega_{p h} d \cos \theta_{f}}=\frac{Z_{\text {eff }}{ }^{2} e^{2}}{2 \pi c^{5}} \frac{w_{p h} E_{i}}{m^{2} k_{i}}\left\{p\left(k_{i}, k_{f}\right) \frac{d p^{*}\left(k_{i}, k_{f}, \Omega_{f}\right)}{d \cos \theta_{f}}+\text { h.e. }\right\} .
\end{aligned}
$$

\section{H. Multipolar Approach}

Let us find the angular probability in eqs. (50) at the first values $l_{f}=1$ and $l_{p h}=1$. Following eqs. (14), (32) and (15), we have:

$$
\begin{aligned}
\tilde{p}_{1}\left(k_{i}, k_{f}\right) & =\left.\sqrt{2 \pi} \cdot(-i)^{l_{p h}} \sqrt{2 l+1} \cdot\left(\tilde{p}_{l_{p h}}^{M}-i \tilde{p}_{p_{p h}}^{E}\right)\right|_{l_{p h}=1} \\
& =-\sqrt{6 \pi}\left(i \tilde{p}_{1}^{M}+\tilde{p}_{1}^{E}\right), \\
\frac{d \tilde{p}_{1}\left(k_{i}, k_{f}\right)}{\sin \theta d \theta} & =\left.\sqrt{2 \pi} \cdot(-i)^{l_{p h}} \sqrt{2 l_{p h}+1} \cdot\left(\frac{d \tilde{p}_{l_{p h}}^{M}}{\sin \theta d \theta}-i \frac{d \tilde{p}_{l_{p h}}^{E}}{\sin \theta d \theta}\right)\right|_{l_{p h}=1} \\
& =-\sqrt{6 \pi}\left\{i \frac{d \tilde{p}_{1}^{M}}{\sin \theta d \theta}+\frac{d \tilde{p}_{1}^{E}}{\sin \theta d \theta}\right\} .
\end{aligned}
$$

Using the found differential electric and magnetic components (36):

$$
\begin{aligned}
& \frac{d \tilde{p}_{1}^{M}}{\sin \theta d \theta}=-\frac{3}{8} \sqrt{\frac{1}{\pi}} \cdot J(1,1) \cdot \sin ^{2} \theta \cos \theta, \\
& \frac{d \tilde{p}_{1}^{E}}{\sin \theta d \theta}=i \frac{1}{8} \sqrt{\frac{2}{\pi}} \cdot J(1,0) \cdot \sin ^{2} \theta+i \frac{1}{8} \sqrt{\frac{1}{\pi}} \cdot J(1,2) \cdot \sin ^{2} \theta\left(1-3 \sin ^{2} \theta\right)
\end{aligned}
$$

and the integral components (37):

$$
\begin{aligned}
& \tilde{p}_{1}^{M}=0, \\
& \tilde{p}_{1}^{E}=i \frac{1}{6} \sqrt{\frac{2}{\pi}} \cdot\left(J(1,0)-\frac{7}{10} \sqrt{2} \cdot J(1,2)\right),
\end{aligned}
$$

from eq. (51) we obtain:

$$
\begin{aligned}
\tilde{p}_{1}\left(k_{i}, k_{f}\right) & =-i \sqrt{\frac{1}{3}} \cdot\left(J(1,0)-\frac{7}{10} \sqrt{2} \cdot J(1,2)\right), \\
\frac{d \tilde{p}_{1}\left(k_{i}, k_{f}\right)}{\sin \theta d \theta} & =i \frac{\sqrt{6}}{8} \cdot\{3 J(1,1) \cdot \cos \theta-\sqrt{2} J(1,0)- \\
& -J(1,2) \cdot\left(1-3 \sin ^{2} \cdot\right\} \sin ^{2} \theta .
\end{aligned}
$$
(43):

Now we find the relative angular probability from eq.

$$
\begin{aligned}
\frac{d W_{1}^{E 1+M 1}\left(\theta_{f}\right)}{d \Omega_{p h} d \cos \theta_{f}} & =\frac{Z_{\text {eff }}{ }^{2} \hbar e^{2}}{8 \pi c^{3}} \frac{w_{f i}}{m^{2}}\left\{\left(J(1,0)-\frac{7}{10} \sqrt{2} \cdot J(1,2)\right) \times\right. \\
& \times\left(J^{*}(1,0)+\frac{1}{\sqrt{2}} J^{*}(1,2) \cdot\left(1-3 \sin ^{2} \theta\right)-\right. \\
& \left.\left.-\frac{3}{\sqrt{2}} J^{*}(1,1) \cdot \cos \theta\right)+ \text { h.e. }\right\} \cdot \sin ^{2} \theta
\end{aligned}
$$

and the absolute angular probability from eq. (50):

$$
\begin{aligned}
\frac{d P_{1}^{E 1+M 1}\left(\theta_{f}\right)}{d \Omega_{p h} d \cos \theta_{f}} & =\frac{Z_{\text {eff }}{ }^{2} e^{2}}{8 \pi c^{5}} \frac{w_{f i}}{m^{2}} \frac{E_{i}}{k_{i}} \times \\
& \times\left\{\left(J(1,0)-\frac{7}{10} \sqrt{2} \cdot J(1,2)\right) \times\right. \\
& \times\left(J^{*}(1,0)+\frac{1}{\sqrt{2}} J^{*}(1,2) \cdot\left(1-3 \sin ^{2} \theta\right)-\right. \\
& \left.\left.-\frac{3}{\sqrt{2}} J^{*}(1,1) \cdot \cos \theta\right)+ \text { h.e. }\right\} \cdot \sin ^{2} \theta .
\end{aligned}
$$




\section{Nucleus- $\alpha$-Particle Potential}

Knowledge of the $\alpha$-nucleus interaction potential is a key for the analysis of various reactions between $\alpha$-particle and nuclei. The nucleus-nucleus interaction potential consists of both Coulomb repulsion and nuclear attraction parts. These two parts form a barrier at small distances between $\alpha$ particle and nuclei. The Coulomb component of the potential is well-known. In contrast, the nuclear part of the potential is less well-defined. There are many different approaches to the nuclear part of the interaction potential between $\alpha$-particle and nuclei [29-39]. $\alpha$-decay [31, 33-35, 38] and various scattering $[29,30,32,36,37]$ data are used for evaluation of the $\alpha$-nucleus potential. Potentials [29-38, 32] evaluated for the same colliding system using different approaches differ considerably, and it is difficult to describe various reaction data from many nuclei at energies deeply below and around the barrier by only one type of such a potential with a good accuracy (e.g., the IAEA Reference Input Parameter Library [39]).

However, $\alpha$-decay half-lives depend strongly on the $\alpha$ nucleus potential and they are used as a main test of the shape of the studied $\alpha$-nucleus potential for the $\alpha$-decay. At present, in two-body approach (for example, see Refs. [18, $24,40]$ ), the found potential reproduces the measured halflives in a huge region of numbers $A_{\mathrm{d}}$ and $Z_{\mathrm{d}}$ of the nuclei. But in several other papers [41-47], the alpha-decay halflives were calculated from more realistic potentials where the ratio between the experimental and calculated half-lives has been interpreted as preformation factor of the alphaparticle in the decaying nucleus. By such approach, it becomes more suitable to describe properties of bound states [48]. Shapes of the barriers of such two types of the $\alpha$ nucleus potential differ, and one hope that further study of the bremsstrahlung processes accompanying the $\alpha$-decay were allowed us to find the most suitable one.

But, at present stage, to describe the interaction between the $\alpha$-particle and the daughter nucleus the potentialhas been used in the following general form (see Refs. [22, 23]):

$V(r, \theta, l, Q)=v_{C}(r, \theta)+v_{N}(r, \theta, Q)+v_{l}(r)$,

where the Coulomb $v_{C}(r, \theta)$, nuclear $v_{N}(r, \theta, Q)$ and centrifugal $v_{l}(r)$ components are

$v_{C}(r, \theta)=\left\{\begin{array}{cc}\frac{2 Z e^{2}}{r}\left(1+\frac{3 R^{2}}{5 r^{2}} \beta_{2} Y_{20}(\theta)\right), & \text { for } r \geq r_{m}, \\ \frac{2 Z e^{2}}{r_{m}}\left\{\frac{3}{2}-\frac{r^{2}}{2 r_{m}^{2}}+\frac{3 R^{2}}{5 r_{m}^{2}}\left(2-\frac{r^{3}}{r_{m}^{3}}\right) \beta_{2} Y_{20}(\theta)\right\}, & \text { for } r<r_{m},\end{array}\right.$

$v_{N}(r, \theta, Q)=\frac{V\left(A_{\mathrm{d}}, Z_{\mathrm{d}}, Q\right)}{1+\exp \frac{r-r_{m}(\theta)}{d}}, \quad v_{l}(r)=\frac{l(l+1)}{2 m r^{2}}$.

For determination of parameters of the Coulomb and nuclear components An approach proposed in Ref. [18] has been used (see relations (14), (16)-(19) in this paper):

$$
\begin{aligned}
V\left(A_{\mathrm{d}}, Z_{\mathrm{d}}, Q\right)= & -\left(30.275-0.45838 Z_{\mathrm{d}} / A_{\mathrm{d}}{ }^{1 / 3}+\right. \\
& +58.270 I-0.24244 Q), \\
R= & R_{p}\left(1+3.0909 / R_{p}^{2}\right)+0.1243 t, \\
R_{p}= & 1.24 A_{\mathrm{d}}^{1 / 3}\left(1+1.646 / A_{\mathrm{d}}-0.191 I\right), \\
t= & I-0.4 A_{\mathrm{d}} /\left(A_{\mathrm{d}}+200\right), \\
d= & 0.49290, \\
I= & \left(A_{\mathrm{d}}-2 Z_{\mathrm{d}}\right) / A_{\mathrm{d}},
\end{aligned}
$$

and:

$$
\begin{aligned}
& r_{m}(\theta)=1.5268+R(\theta), \\
& R(\theta)=R\left(1+\beta_{2} Y_{20}(\theta)\right) .
\end{aligned}
$$

Here, $Q$ is the $Q_{\alpha}$-value for the $\alpha$-decay, $R$ is the radius of the daughter nucleus, $V\left(A_{\mathrm{d}}, Z_{\mathrm{d}}, Q\right)$ is the strength of the nuclear component, $r_{m}$ is the effective radius of the nuclear component, $d$ is the parameter of the diffuseness, $Y_{20}(\theta)$ is the spherical harmonic function of the second order, $\theta$ is the angle between the direction of the leaving $\alpha$-particle and the axis of the axial symmetry of the daughter nucleus, $\beta_{2}$ is the parameter of the quadruple deformation of the daughter nucleus. Values of $R, R(\theta), r_{m}, r, d$ are used in fermi, whereas $Q_{\alpha}, V(r, \theta, l, Q), V\left(A_{\mathrm{d}}, Z_{\mathrm{d}}, Q\right)$ are used in $\mathrm{MeV}$.

\section{CALCULATIONS AND ANALYSIS}

\section{A. Bremsstrahlung Spectra for ${ }^{210} \mathrm{Po},{ }^{214} \mathrm{Po}$ and ${ }^{226} \mathrm{Ra}$ : Comparison Theory and Experiments}

With a purpose to estimate efficiency of the definition of the angular absolute probability of the photon emission and accuracy of the model, I shall calculate the spectra for the ${ }^{210} \mathrm{Po}$, ${ }^{214} \mathrm{Po}$ and ${ }^{226} \mathrm{Ra}$ nuclei has been calculated and compared with experimental data for these nuclei. Here, the bremsstrahlung probability was calculated by eq. (54) at $l=0$ in the calculation of $p(w, \vartheta)$. The angle $\theta$ between the directions of the $\alpha$ particle motion (with possible tunneling) and the photon emission is $90^{\circ}$. The nucleus- $\alpha$-particle potential is defined in eqs. (55)-(57), its parameters are defined in eqs. (58)-(60). $Q_{\alpha}$-value is $+5.439 \mathrm{MeV}$ for ${ }^{210} \mathrm{Po},+7.865 \mathrm{MeV}$ for ${ }^{214} \mathrm{Po},+4.904 \mathrm{MeV}$ for ${ }^{226} \mathrm{Ra}$, according to Ref. [24] (see p. 63). The best result in agreement between theory and experiment have been obtained for the ${ }^{214}$ Po nucleus (Fig. 1a), here there is no any normalization of the calculated curve relatively experimental data). From the figure, one can see that for this nucleus, the calculated spectrum by the proposed approach is in enough good agreement with the experimental data [22] inside the region from $100 \mathrm{keV}$ up to $750 \mathrm{keV}$. The calculated absolute probabilities of the bremsstrahlung in $\alpha$-decay of the ${ }^{210} \mathrm{Po}$ and ${ }^{226} \mathrm{Ra}$ nuclei and experimental data in Refs. [4] and [23] for these nuclei are presented in Fig. (1b, c). In both nuclei for low energies of the photons emitted, the calculated spectra are located below experimental data, but for energies from $350 \mathrm{keV}$ and higher, a good agreement has obtained between theory and experiment. 
Agreement between spectrum of the absolute bremsstrahlung probability calculated by the multipole model for the ${ }^{226}$ Ra nucleus (see solid red line 3 in Fig. (1)) and experimental data [23] for this nucleus (see scatter 1, in Fig. (1)) looks not so well as agreement between spectrum of the normalized bremsstrahlung probability calculated in Ref. [23] by another model (see dash-dot green line 2 in Fig. (1)) and the experimental data. But, in the angular description of the emission of photon during $\alpha$-decay the multipolar model looks the most accurate and motivated from the physical point of view among variety of different angular models and approaches. In particular, if in eq. (53), for the relative probability, and in eq. (54) for the absolute probability restricting ourselves by the first most important integral $J(1,0)$ only in a comparison with other two integrals $J(1,1)$ and $J(1,2)$ then (without normalization constant) the spectrum in definition of the model in Ref. [23] has been obtained explicitely where expansion of the wave function of photons by spherical waves was used. As a result, applying such restriction and further normalization (which was used in Ref. [23]) by the multipolar model, spectrum 2 (see green dash-dot line, Fig. (1)) has been obtained exactly. Now a difference between old spectrum (see green dash-dot line 2, Fig. (1)) from Ref. [23] and new spectrum (see red solid line 3, Fig. (1)) obtained by the multipolar model is explained by taking into account the non-zero magnetic components $p_{l_{p h}}^{M}$ in the total matrix element in eqs. (13) or $(51)(J(1,2)$ is smaller than $J(1,0)$ by $1-2$ orders inside the energy region of the photons emitted from $50 \mathrm{keV}$ up to $1 \mathrm{MeV}$ practically). One can stress that in the angular study of the emission of photon the dipole approximation used by T. Papenbrock and G. F. Bertsch in Ref. [14] and by other researchers in further papers (for example, see Ref. $[3,5,17]$ ), which are based on such approximation, restricts the emission of photons to isotropic (prove of this fact can be found in Appendix of Ref. [19]). The wave function $\psi_{f}(\mathbf{r})$ of the $\alpha$-decaying system after the emission of photon has no any angular information about this photon. The angular integral in such dipole approach has no angular wave function of photons also. By other words, the dipole approach cannot give any useful information about angular distribution of the emission of photons. In particular, by such a reason attempts to explain a difference between experimental data [49] (obtained for the angle $90^{\circ}$ ) and experimental data $[50,51]$ (obtained for the angle $25^{\circ}$ ) by the different angle values on the basis of the dipole model has no sense (also see discussions in Refs. [52, 53]). Such motivations confirm effectiveness of the multipolar model in the calculations of the angular absolute probability, and the multipolar model has been used as needed, and perspective basis in further construction of unified formula of the bremsstrahlung probability in the $\alpha$-decay are presented below.

\section{B. Formula of the Bremsstrahlung Probability in the $\alpha$ - Decay}

Analyzing the Th isotopes, It was observed that trend of the bremsstrahlung spectrum depends on $Q_{\alpha}$-value directly.
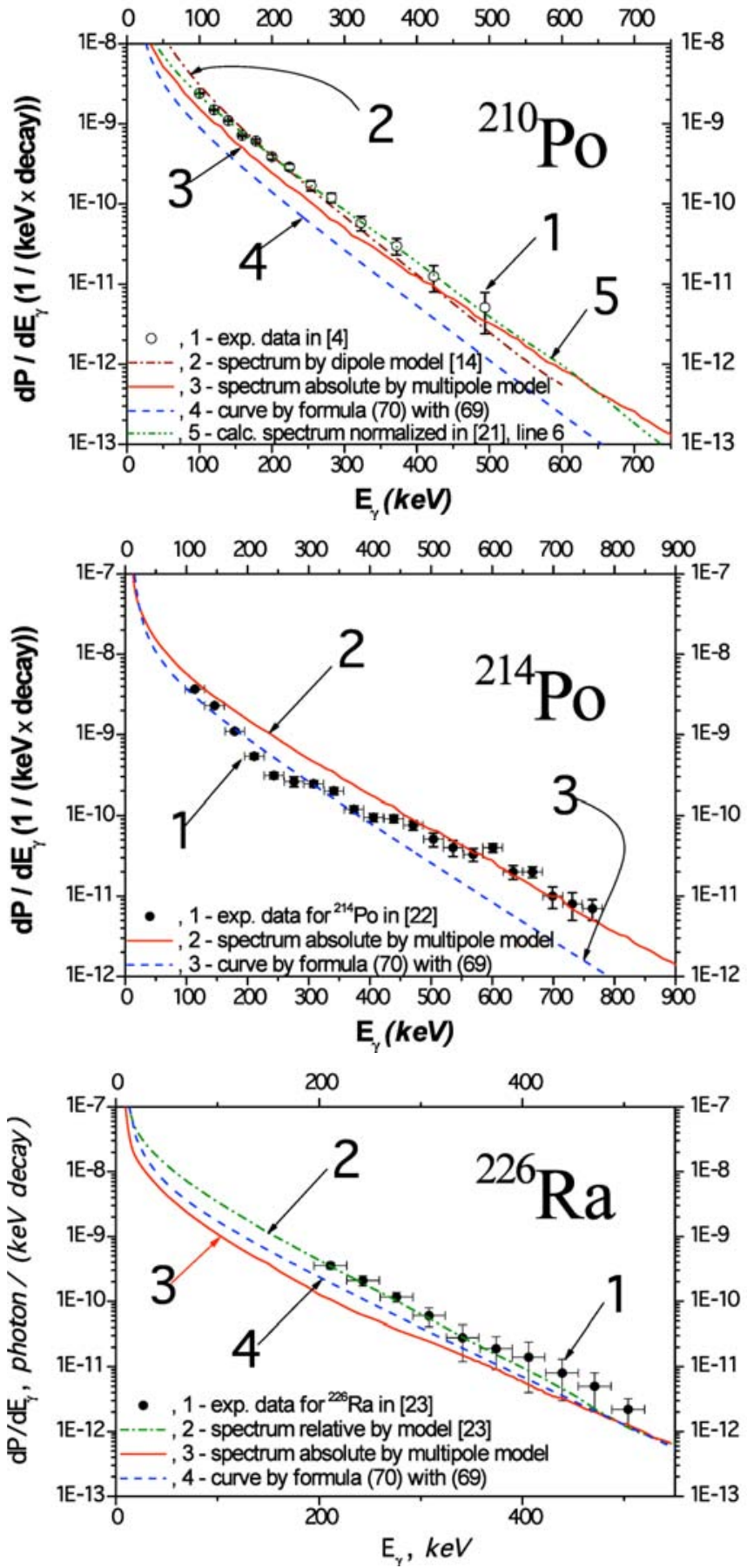

Fig. (1). The bremsstrahlung probability in the $\alpha$-decay of the ${ }^{214} \mathrm{Po},{ }^{210} \mathrm{Po}$ and ${ }^{226} \mathrm{Ra}$ nuclei: the absolute probability calculated by the multipolar model (red solid line), experimental data (scatter, data [22] for ${ }^{214} \mathrm{Po}$, data [4] for ${ }^{210} \mathrm{Po}$ and data [23] for ${ }^{226} \mathrm{Ra}$ ) and curve calculated by formula (70) with (69) (dash blue line). Description by the formula (70) with (69) can be improved further, if to pass from the linear dependence (64) of the probability on $A_{\mathrm{d}}$ and $Z_{\mathrm{d}}$ to harmonic one.

In Ref. [17], dependence of the bremsstrahlung probability on the electrical charge of the daughter nucleus was analyzed. Calculating the probability for nuclei with different mass numbers at the same charge, one can see that this probability depends rather on combination of numbers of protons 
and neutrons of the nucleus than its electric charge (at the same $Q_{\alpha}$ ). So, The conclusion has been drawn on direct dependence of the bremsstrahlung probability on the effective charge $Z_{\text {eff }}$ (at the first time) Formulas (2) confirm such dependence explicitly. But it is interesting to know as to how much the bremsstrahlung depends on $Q_{\alpha}$-value quantitatively and the most important other parameters of the $\alpha$-decay. It should be convenient to compose a unified formula, which calculates the bremsstrahlung probability in the $\alpha$-decay of arbitrary nucleus on the basis of all these parameters directly. But, whether is it possible to describe the bremsstrahlung spectra for all different nuclei by only one formula in general? Whether is it possible to describe the bremsstrahlung spectrum for only one arbitrary nucleus with very high accuracy inside the energy region of the photon emitted used in experiments?

At present day, there are experimental data of the bremsstrahlung photons accompanying $\alpha$-decay for only four nuclei: ${ }^{210} \mathrm{Po},{ }^{214} \mathrm{Po},{ }^{226} \mathrm{Ra},{ }^{244} \mathrm{Cm}$. Here, errors in experimental data for ${ }^{244} \mathrm{Cm}$ to be larger, and therefore, three other nuclei ${ }^{210} \mathrm{Po},{ }^{214} \mathrm{Po}$, and ${ }^{226} \mathrm{Ra}$ are analyzed mainly in literature. By that reason, such supposed formula has been tried to constructed for the $\alpha$-decay of arbitrary nucleus, and then test it on the basis of the model, which as suppose should give the most reliable calculated spectra for arbitrary nucleus with arbitrary energy of $\alpha$-decay. As such a model, the multipole model is used, which is presented before. It is supposed that this model this model is the most accurate in calculations of the angular bremsstrahlung spectra in the $\alpha$-decay. This model has been tested on the basis of experimental data for the ${ }^{210} \mathrm{Po},{ }^{214} \mathrm{Po},{ }^{226} \mathrm{Ra}$ nuclei. If some approximations were used then the results published previously were obtained by our group or by dipole approach (with normalization on the experimental data) and using corresponding shapes of the $\alpha$ nucleus potential.

At first, let us restrict ourselves by only one nucleus and try to write such formula for it. After preliminary estimations of the spectra for different nuclei, the following form has been proposed:

$$
\begin{aligned}
& \ln \left(\frac{d P_{\text {param }}\left(w ; a_{0} \ldots a_{4}, n_{1} \ldots n_{4}\right)}{d \Omega_{p h} d \cos \theta_{f}}\right)= \\
& =\ln \left(\frac{e^{2}}{8 \pi c^{5}} \frac{Z_{\text {eff }}{ }^{2} E_{i}}{m^{2} k_{i}}\right)+a_{0}-a_{1} w^{n_{1}}+ \\
& +\frac{a_{2}}{w^{n_{2}}}+\frac{a_{3}}{w^{n_{3}}}+\frac{a_{4}}{w^{n_{4}}},
\end{aligned}
$$

where $a_{0} \ldots a_{4}$ and $n_{1} \ldots n_{4}$ are unknown constants which do not depend on energy of the photon emitted and are changed for the different nuclei. These constants reflect "structure" of the $\alpha$-decay for the studied nucleus. Therefore, they should depend on $Q_{\alpha}, Z_{\mathrm{eff}}, Z_{\mathrm{d}}$ and $A_{\mathrm{d}}$ of this nucleus. In further determination of the unknown parameters $a_{0} \ldots a_{4}$ and $n_{1} \ldots n_{4}$, the angle $\theta_{\mathrm{f}}$ equal to $90^{\circ}$ has been used. There are two rea- sons for such a choice: (1) Experimental data [49, 52] are obtained at this angle. (2) The spectra should be maximal, and therefore, possible error in determination of $a_{0} \ldots a_{4}$ and $n_{1} \ldots n_{4}$ should be minimal.

With a purpose to find parameters $a_{0} \ldots a_{4} \quad n_{1} \ldots n_{4}$ for the selected nucleus, the following characteristic are introduced:

$$
\begin{aligned}
\sigma\left(a_{i}, n_{i}\right) & =\sqrt{\frac{\int_{w_{\min }}^{w_{\max }}\left(\Delta P\left(w, a_{i}, n_{i}\right)\right)^{2} d w}{w_{\text {max }}-w_{\text {min }}}}, \\
\Delta P\left(w ; a_{i}, n_{i}\right) & =\ln \left(\frac{d P_{\text {model }}(w)}{d \Omega_{p h} d \cos \theta_{f}}\right)- \\
& -\ln \left(\frac{d P_{\text {param }}\left(w ; a_{0} \ldots a_{4}, n_{1} \ldots n_{4}\right)}{d \Omega_{p h} d \cos \theta_{f}}\right),
\end{aligned}
$$

where $d P_{\text {model }}$ and $d P_{\text {param }}$ are the bremsstrahlung probabilities calculated by the multipolar model and by the formula (61), correspondingly. The $\sigma$ at selected set of parameters $a_{0} \ldots a_{4}$ and $n_{1} \ldots n_{4}$ is smaller, the curve $d P_{\text {param }}$ obtained by formula (61) is closer to the spectrum $d P_{\text {model }}$ calculated by the multipolar model i.e. the most accurate description of the bremsstrahlung spectrum for the studied nucleus by formula (61) should be obtained at such choice of the parameters $a_{0} \ldots a_{4}$ and $n_{1} \ldots n_{4}$ when $\sigma$ is minimal. These parameters have been found requiring $\sigma$ being minimal. For convenience, this approach has been called for determination of parameters for the selected nucleus as a method of minimization.

In Ref. [54] (see p. D395), authors reported about experimental measurements of the bremsstrahlung accompanying $\alpha$-decay of the ${ }^{228} \mathrm{Th}$ nucleus and some perspectives. Of course, such experiments will be able to enlarge experimental data existed in this topic. With a purpose to reinforce such investigations, isotopes of this nucleus have been used in the first calculations. Thus, using the method of minimization, for the ${ }^{218} \mathrm{Th}$ nucleus with $Q$-value equal to $9.881 \mathrm{MeV}$, the following values have been obtained $\left(w_{\min }=50 \mathrm{keV}\right.$ and $w_{\max }=900 \mathrm{keV}$ are used; choice of the ${ }^{218} \mathrm{Th}$ nucleus from different isotopes Th is made because it gives the best convergence in calculations of the bremsstrahlung spectra by the multipolar model):

$$
\begin{array}{llll}
n_{1}=1, & n_{2}=0.5, & n_{3}=1, & n_{4}=2, \\
a_{0}=10.8, & a_{1}=0.007, & a_{2}=10, & a_{3}=10,
\end{array}
$$

In Fig. (2a), comparison between the curve obtained by the formula (61) with parameters (63) (see dash-dot red line in this figure) and the spectrum calculated by the multipole model (see solid blue line in this figure) is presented. Here, one can see that agreement between such two lines looks extremely accurate up to $1 \mathrm{MeV}$ ! This confirms that the spectrum obtained by formula (61) with parameters (63) is very close to the result obtained by the multipolar model for the $\alpha$-decay of the ${ }^{218} \mathrm{Th}$ nucleus with Q-value $9.881 \mathrm{MeV}$. From here, it is concluded that the bremsstrahlung probability for arbitrary 
one nucleus can be approximated by formula (61) with very high accuracy inside the energy region up to $1 \mathrm{MeV}$. Estimations of parameters for other nuclei show that it is possible to describe the bremsstrahlung spectra with enough high accuracy for different nuclei using different values of the $a_{0}$ and $a_{1}$ parameters only, while the $n_{1} \ldots n_{4}$ parameters and even the $a_{2}, a_{3}, a_{4}$ parameters are fixed.

(a)

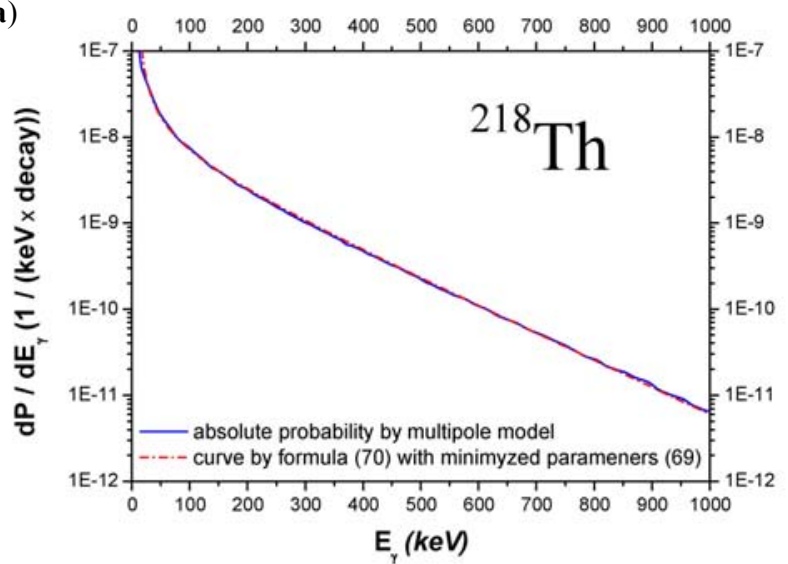

(b)

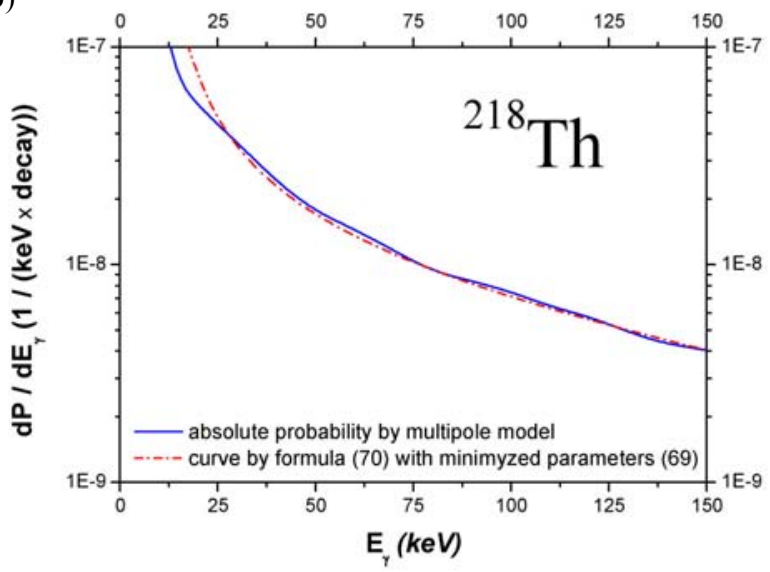

Fig. (2). Comparison between the bremsstrahlung probability $d P_{\text {model }}$ for the ${ }^{218} \mathrm{Th}$ nucleus calculated by the multipolar model (blue solid curve) and curve $d P_{\text {param }}$ for this nucleus calculated by formula (70) with parameters (69) (red dash dot curve): (a) one can see that after minimization this curve describes the spectrum very accurately up to $1 \mathrm{MeV}$, (b) the difference between two spectra is presented in larger scale up to $150 \mathrm{keV}$.

This paper defines the $n_{1} \ldots n_{4}, a_{2}, a_{3}$ and $a_{4}$ parameters for the different nuclei by eqs. (63). Let us find how $a_{0}$ and $a_{1}$ can be described. Assuming dependence of $a_{0}$ and $a_{1}$ on $Q, A_{\mathrm{d}}$ and $Z_{\mathrm{d}}$ to be linear, the following formula has been proposed:

$$
\begin{aligned}
& a_{0}\left(Q, A_{\mathrm{d}}, Z_{\mathrm{d}}\right)=b_{00}+b_{01} Q+b_{02} A_{\mathrm{d}}+b_{03} Z_{\mathrm{d}}, \\
& a_{1}\left(Q, A_{\mathrm{d}}, Z_{\mathrm{d}}\right)=b_{10}+b_{11} Q+b_{12} A_{\mathrm{d}}+b_{13} Z_{\mathrm{d}},
\end{aligned}
$$

where new unknown parameters $b_{0 i}$ and $b_{1 i}(i=0,1,2,3)$ have been introduced and they do not depend on $Q, A_{\mathrm{d}}$ and $Z_{\mathrm{d}}$. Now a problem consists in finding of the unknown parameters $b_{0 i}$ and $b_{1 i}$.
The simplest way is to find $b_{01}$ and $b_{11}$. For the determination of the unknown parameters $b_{01}$ and $b_{11}$, a nucleus with two different $Q$-values is needed. Let us consider the ${ }^{228}$ Th nucleus. Two bremsstrahlung spectra has been calculated for this nucleus on the basis of the multipolar model at two different $Q$-values (I use: $Q_{1}=5.555 \mathrm{MeV}$, and $Q_{2}=10$ $\mathrm{MeV}$ ), and then the $a_{0}$ and $a_{1}$ parameters (different for two $Q$-values) have been obtained using the method of minimization above. Results of such calculations are presented in Table 1 , in the first two strings with numbers 1 and 2 . Using data for this nucleus with such two $Q$-values, from eq. (64) one can write:

$a_{0}\left(Q_{1}\right)-a_{0}\left(Q_{2}\right)=b_{01}\left(Q_{1}-Q_{2}\right)$,
$a_{1}\left(Q_{1}\right)-a_{1}\left(Q_{2}\right)=b_{11}\left(Q-Q_{2}\right)$.

Dividing these equations on $Q_{1}-Q_{2}$, we find:

$b_{01}=\frac{a_{0}\left(Q_{1}\right)-a_{0}\left(Q_{2}\right)}{Q_{1}-Q_{2}}, \quad b_{11}=\frac{a_{1}\left(Q_{1}\right)-a_{1}\left(Q_{2}\right)}{Q_{1}-Q_{2}}$.

Thus, values for parameters $b_{01}, b_{11}$ have been obtained.

With a purpose to find the next four unknown parameters $b_{02}, b_{03}, b_{12}, b_{13}$, it needs to consider four different nuclei with the different $A_{\mathrm{d}}, Z_{\mathrm{d}}$ numbers at the same $Q$-value. One can suppose that accuracy in such calculations could be achieved as high as possible, if in addition to the previous nucleus two other nuclei with the largest difference between $A_{\mathrm{d}}$ and $Z_{\mathrm{d}}$ were used. Let us use Table 1 in Ref. [24] where half-lives in the large region of $\alpha$-decaying nuclei are presented. From here ${ }^{106} \mathrm{Te}$ and nucleus with $A_{\mathrm{p}}=266, Z_{\mathrm{p}}=109$ is selected. the bremsstrahlung spectra at $Q_{\alpha}$-value equals to $10 \mathrm{MeV}$ has been calculated using the multipolar model, and then $a_{0}$ and $a_{1}$ has been found for them using the minimization method. Results are presented in Table 1, in the next two strings with numbers 3 and 4. From eq. (64), we write:

$$
\begin{aligned}
& a_{0}^{(3)}-a_{0}^{(2)}=b_{02}\left(A_{3}-A_{2}\right)+b_{03}\left(Z_{3}-Z_{2}\right), \\
& a_{0}^{(4)}-a_{0}^{(3)}=b_{02}\left(A_{4}-A_{3}\right)+b_{03}\left(Z_{4}-Z_{3}\right), \\
& a_{1}^{(3)}-a_{1}^{(2)}=b_{12}\left(A_{3}-A_{2}\right)+b_{13}\left(Z_{3}-Z_{2}\right), \\
& a_{1}^{(4)}-a_{1}^{(3)}=b_{12}\left(A_{4}-A_{3}\right)+b_{13}\left(Z_{4}-Z_{3}\right),
\end{aligned}
$$

and from here we find:

$$
\begin{aligned}
& b_{02}=\frac{\left(a_{0}^{(3)}-a_{0}^{(2)}\right)\left(Z_{4}-Z_{3}\right)-\left(a_{0}^{(4)}-a_{0}^{(3)}\right)\left(Z_{3}-Z_{2}\right)}{\left(A_{3}-A_{2}\right)\left(Z_{4}-Z_{3}\right)-\left(A_{4}-A_{3}\right)\left(Z_{3}-Z_{2}\right)}, \\
& b_{03}=\frac{\left(a_{0}^{(4)}-a_{0}^{(3)}\right)\left(A_{3}-A_{2}\right)-\left(a_{0}^{(3)}-a_{0}^{(2)}\right)\left(A_{4}-A_{3}\right)}{\left(A_{3}-A_{2}\right)\left(Z_{4}-Z_{3}\right)-\left(A_{4}-A_{3}\right)\left(Z_{3}-Z_{2}\right)}, \\
& b_{12}=\frac{\left(a_{1}^{(3)}-a_{1}^{(2)}\right)\left(Z_{4}-Z_{3}\right)-\left(a_{1}^{(4)}-a_{1}^{(3)}\right)\left(Z_{3}-Z_{2}\right)}{\left(A_{3}-A_{2}\right)\left(Z_{4}-Z_{3}\right)-\left(A_{4}-A_{3}\right)\left(Z_{3}-Z_{2}\right)}, \\
& b_{13}=\frac{\left(a_{1}^{(4)}-a_{1}^{(3)}\right)\left(A_{3}-A_{2}\right)-\left(a_{1}^{(3)}-a_{1}^{(2)}\right)\left(A_{4}-A_{3}\right)}{\left(A_{3}-A_{2}\right)\left(Z_{4}-Z_{3}\right)-\left(A_{4}-A_{3}\right)\left(Z_{3}-Z_{2}\right)}
\end{aligned}
$$


Table 1. Parameters $a_{0}$ and $a_{1}$ for ${ }^{228} \mathrm{Th},{ }^{106}$ Te and Nucleus with $\mathrm{A}_{\mathbf{d}}=266$ and $\mathrm{Z}_{\mathbf{d}}=109\left(a_{0}^{(\mathrm{min})}\right.$ and $a_{1}^{(\mathrm{min})}$ are Parameters Calculated by Method of Minimization, $a_{0}^{(\text {param })}$ and $a_{1}^{(\text {param })}$ are Parameters Calculated by Formula (70))

\begin{tabular}{|c|c|c|c|c|c|c|c|c|c|}
\hline \multicolumn{9}{|c|}{$\alpha$-Decay Data } & \multicolumn{3}{c|}{ Parameters } \\
\hline No. & $\mathbf{A}_{\mathbf{d}}$ & $\mathrm{A}_{\mathrm{d}}^{1 / 3}$ & $\mathbf{Z}_{\mathbf{d}}$ & $\mathbf{Z}_{\text {eff }}$ & $\mathbf{Q}_{\alpha}, \mathbf{M e V}$ & $a_{0}^{(\min )}$ & $a_{0}^{(\text {(param })}$ & $a_{1}^{(\text {min })}$ & $a_{1}^{(\text {(param })}$ \\
\hline \hline 1 & 224 & 6.073177 & 88 & 0.42105 & 5.555 & 10.2 & 10.20083 & 0.0154 & 0.01531749 \\
\hline 2 & 224 & 6.073177 & 88 & 0.42105 & 10.0 & 11.2 & 11.20020 & 0.0069 & 0.00681732 \\
\hline 3 & 102 & 4.672328 & 50 & 0.03774 & 10.0 & 6.3 & 6.30084 & 0.00475 & 0.00440210 \\
\hline 4 & 262 & 6.398827 & 107 & 0.36090 & 10.0 & 10.9 & 10.90000 & 0.008 & 0.00799993 \\
\hline
\end{tabular}

$b_{00}=a_{0}(Q, A, Z)-b_{01} Q-b_{02} A-b_{03} Z$

$b_{10}=a_{1}(Q, A, Z)-b_{11} Q-b_{12} A-b_{13} Z$.

Using data of Table $\mathbf{1}$, we calculate unknown $b_{0 i}, b_{1 i}$ :

$b_{00}=4.60202, \quad b_{10}=0.0204108$,

$b_{01}=0.22497, \quad b_{11}=-0.0019123$,

$b_{02}=0.11956, \quad b_{12}=1.086956 \cdot 10^{-6}$,

$b_{03}=-0.25492, \quad b_{13}=6.0068649 \cdot 10^{-5}$.

So, we have found the following dependence of $a_{0}$ and $a_{1}$ on $Q, A_{\mathrm{d}}$ and $Z_{\mathrm{d}}(Q$ is used in $\mathrm{MeV}$ ) have been found:

$$
\begin{aligned}
a_{0}\left(Q, A_{\mathrm{d}}, Z_{\mathrm{d}}\right)= & 4.60202+0.22497 \cdot Q+ \\
& +0.11956 \cdot A_{\mathrm{d}}-0.25492 \cdot Z_{\mathrm{d}}, \\
a_{1}\left(Q, A_{\mathrm{d}}, Z_{\mathrm{d}}\right)= & 0.0204108-0.0019123 \cdot Q+ \\
& +1.086956 \cdot 10^{-6} \cdot A_{\mathrm{d}}+ \\
& +6.0068649 \cdot 10^{-5} \cdot Z_{\mathrm{d}}
\end{aligned}
$$

and the bremsstrahlung formula (61) has transformed into such:

$$
\begin{aligned}
& \ln \left(\frac{d P_{1}^{E 1+M 1}\left(w, \theta_{f}=90^{\circ}\right)}{d \Omega_{p h} d \cos \theta_{f}}\right)= \\
& =\ln \left(\frac{e^{2}}{8 \pi c^{5}} \frac{Z_{\mathrm{eff}}{ }^{2} E_{i}}{m^{2} k_{i}}\right)+a_{0}-a_{1} w+\frac{10}{\sqrt{w}}+\frac{10}{w}+\frac{1}{w^{2}} .
\end{aligned}
$$

For 4 studied nuclei, the difference between the values of $a_{0}^{(\min )}$ and $a_{1}^{(\mathrm{min})}$ parameters has been obtained by the method of minimization, and the values of the $a_{0}^{\text {(param) }}$ and $a_{1}^{\text {(param) }}$ parameters calculated by formula (70) less then 1 percent. i.e. we have described the bremsstrahlung spectra inside the energy region up to $1 \mathrm{MeV}$ for four different nuclei (with such long maximal distance between their numbers $A_{\mathrm{d}}$ ) with very good accuracy by only one, this formula with parameters calculated only on the basis of values $A_{\mathrm{d}}, Z_{\mathrm{d}}, Q_{\alpha}$. But, unfortunately, it turns out that description of the bremsstrahlung spectra for all nuclei inside region $A_{\mathrm{d}}=$ 107..262 at different $Z_{\mathrm{d}}$ by the formula (70) with parameters (69) is not such good as expected before (maximal error in estimation of the probability is about half of order). How- ever, one can improve such approximation essentially for the "problem" nuclei, if to pass from the linear dependence (64) of the bremsstrahlung probability on the $A_{\mathrm{d}}$ and $Z_{\mathrm{d}}$ values to harmonic one (Fig. 3).

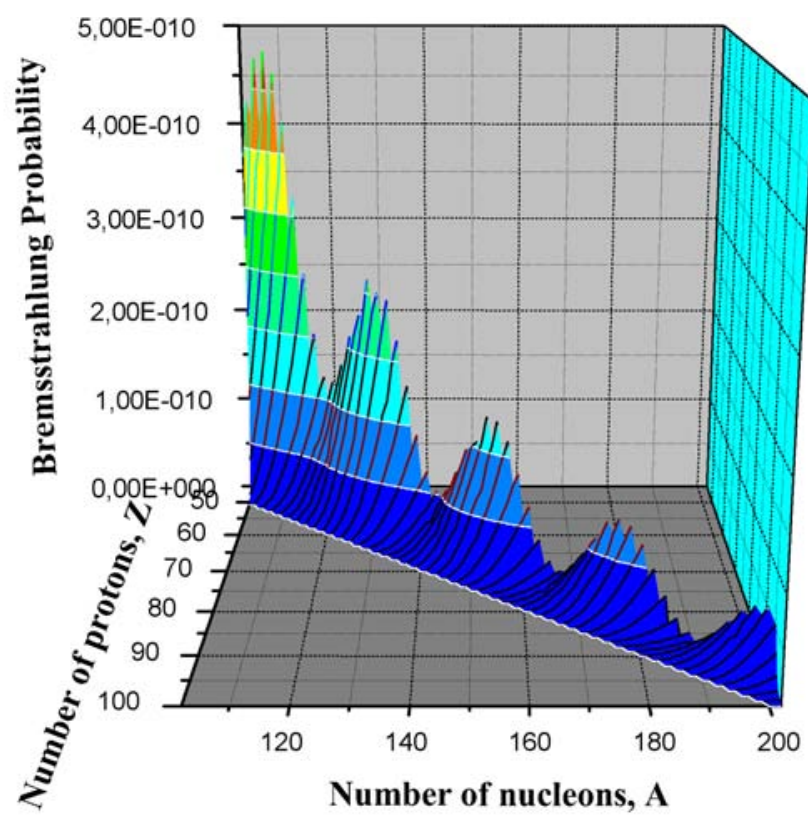

Fig. (3). Harmonic dependence of the bremsstrahlung probability $d P_{\text {model }}$ on different $A_{\mathrm{d}}$ and $Z_{\mathrm{d}}$ values of the nucleus under decay, calculated on the basis of the proposed multipolar model at the same energy of the photon emitted equal to $500 \mathrm{keV}$.

\section{CONCLUSIONS}

The improved multipolar model of the bremsstrahlung accompanying the $\alpha$-decay is presented in the paper. The angular formalism of calculations of the matrix elements is stated in details. Effectiveness of the developed formalism of the model and accuracy of the calculations of the bremsstrahlung spectra are analyzed in their comparison with experimental data for the ${ }^{210} \mathrm{Po},{ }^{214} \mathrm{Po}$ and ${ }^{226} \mathrm{Ra}$ nuclei. Here, note the following.

- The best result has been obtained in agreement between the calculated absolute probability of the bremsstrahlung emission for the ${ }^{214}$ Po nucleus and the newest experimental data [22] for this nucleus inside the region of photons energies from $100 \mathrm{keV}$ up to $750 \mathrm{keV}$ (Fig. 1a), $Q_{\alpha}=+7.865 \mathrm{MeV}$, the angle $\theta$ between the directions of the $\alpha$-particle motion and the photon emission is used $90^{\circ}$ ). 
- The calculated absolute probabilities of the bremsstrahlung emission in $\alpha$-decay of the ${ }^{210}$ Po and ${ }^{226} \mathrm{Ra}$ nuclei for low energies of the photons emitted are located below experimental data [4] and [23], but for the energies from $350 \mathrm{keV}$ and higher a good agreement between the model and the experiment has been obtained (Fig. 1b) and (c), $Q_{\alpha}=+5.439 \mathrm{MeV}$ for ${ }^{210} \mathrm{Po}$ and $Q_{\alpha}=$ $+4.904 \mathrm{MeV}$ for $\left.{ }^{226} \mathrm{Ra}, \theta=90^{\circ}\right)$.

- At the first time, the unified formula of the bremsstrahlung probability during the $\alpha$-decay of the arbitrary nucleus, defined directly on the $Q_{\alpha}$-value and numbers $A_{\mathrm{d}}, Z_{\mathrm{d}}$ of protons and neutrons of this nucleus, has been constructed. Inside the region of the $\alpha$-active nuclei from ${ }^{106} \mathrm{Te}$ up to the nucleus with numbers of nucleons and protons $A_{\mathrm{d}}=262$ and $Z_{\mathrm{d}}=102$ (this region is taken from [24]) with energy of the photons emitted from $50 \mathrm{keV}$ up to $900 \mathrm{keV}$ a good agreement has been achieved between the spectra, obtained on the basis of the multipolar model (where duration of calculations for one selected nucleus is up to 1 day), and the bremsstrahlung spectra obtained on the basis of the proposed formula (where duration of calculations is about some seconds using the same computer).

This formula can be useful for quick estimation of the bremsstrahlung probability during the $\alpha$-decay of any interesting nucleus (without a necessity to study enough complicated quantum models and variety of approximations, to realize enough laborious numerical algorithms of computer calculations of the bremsstrahlung spectra with resolution of divergence problem).

\section{ACKNOWLEDGEMENTS}

The author appreciates Dr. Alexander K. Zaichenko for his assistance in computer realization of numerical methods in calculations of wave functions, Prof. Vladislav S. Olkhovsky for the useful discussions concerning realizations of multiple internal reflections in the problem of $\alpha$-decay and comments of definition of phase times, Prof. Giorgio Giardina for the useful discussions concerning main formalism of the model, dependence of the bremsstrahlung spectra on $Q_{\alpha}$-value of $\alpha$-decay, and aspects to investigate deformed nuclei in this problem, Prof. Volodimir M. Kolomietz for the useful discussions and critical comments concerning the general formalism of the presented model, Dr. Sergei N. Fedotkin for the useful comments concerning definitions of absolute and normalized probabilities of the photons emitted during the $\alpha$-decay, Dr. Alexander G. Magner for useful comments concerning determination of wave function of the $\alpha$-decaying system, Prof. Avazbek K. Nasirov for the interesting discussions concerning different models of $\alpha$-decay and proper choice of the $\alpha$-nucleus potential, Dr. Vladislav Kobychev for the interesting discussions with some analysis concerning behavior of the bremsstrahlung spectra for photon energies close to zero, Dr. Volodymyr Kyrytsya for the useful comments on how to improve the computer integration of matrix elements at large radius $r$ (in asymptotic region) with increasing convergence and keeping accuracy in calculations.

\section{APPENDIX A: CLEBSCH-GORDAN COEFFICIENTS}

Definition of Clebsch-Gordan coefficients has been used, according to Table 1 in Ref. [27] (see p. 317). These coefficients at $j_{b}=1$ and $m_{b}= \pm 1$ are presented in Table 2. Using this Table, I find:

$$
\begin{array}{lll}
(011 \mid 2,-1,1)=0, & (111 \mid 2,-1,1)=0, & (211 \mid 2,-1,1)=\sqrt{\frac{3}{5}} \\
(011 \mid 0,1,1)=\sqrt{\frac{1}{2}}, & (111 \mid 0,1,1)=-\sqrt{\frac{1}{2}}, & (211 \mid 0,1,1)=\sqrt{\frac{1}{10}} \\
(011 \mid 0,-1,-1)=\sqrt{\frac{1}{2}}, & (111 \mid 0,-1,-1)=\sqrt{\frac{1}{2}}, & (211 \mid 0,-1,-1)=\sqrt{\frac{1}{10}} \\
(111 \mid-2,1,-1)=0, & (111 \mid-2,1,-1)=0, & (211 \mid-2,1,-1)=\sqrt{\frac{3}{5}}
\end{array}
$$

\begin{tabular}{|c|c|c|}
\hline & \multicolumn{2}{|c|}{$\left(j_{a} 1 j \mid m_{a} m_{b} m\right)$} \\
\hline & $m_{b}=1$ & $m_{b}=-1$ \\
\hline$j=j_{a}+1$ & $\left(\frac{\left(j_{a}+m\right)\left(j_{a}+m+1\right)}{\left(2 j_{a}+2\right)\left(2 j_{a}+2\right)}\right)^{1 / 2}$ & $\left(\frac{\left(j_{a}-m\right)\left(j_{a}-m+1\right)}{\left(2 j_{a}+1\right)\left(2 j_{a}+2\right)}\right)^{1 / 2}$ \\
\hline$j=j_{a}$ & $-\left(\frac{\left(j_{a}+m\right)\left(j_{a}-m+1\right)}{2 j_{a}\left(j_{a}+1\right)}\right)^{1 / 2}$ & $\left(\frac{\left(j_{a}-m\right)\left(j_{a}+m+1\right)}{2 j_{a}\left(j_{a}+1\right)}\right)^{1 / 2}$ \\
\hline$j=j_{a}-1$ & $\left(\frac{\left(j_{a}-m\right)\left(j_{a}-m+1\right)}{2 j_{a}\left(2 j_{a}+1\right)}\right)^{1 / 2}$ & $\left(\frac{\left(j_{a}+m+1\right)\left(j_{a}+m\right)}{2 j_{a}\left(2 j_{a}+1\right)}\right)^{1 / 2}$ \\
\hline
\end{tabular}

Table 2. Clebsch-Gordan Coefficients at $j_{b}=1$ and $m_{b}= \pm 1$ 
APPENDIX B: CALCULATION OF THE INTEGRAL IN EQ. (25) AND SELECTION RULES IN EQS. (26)

Let us find the integral in the left part of eq. (25). Taking into account different variants of definition of the spherical functions $Y_{l m}(\theta, \phi)$, they are defined according to Ref. [26] (see p. 119, $\left.(28,7)-(28,8)\right)$ :

$$
Y_{l m}(\theta, \phi)=(-1)^{\frac{m+|m|}{2}} i^{l} \sqrt{\frac{2 l+1}{4 \pi} \frac{(l-|m|) !}{(l+|m|) !}} P_{l}^{|m|}(\cos \theta) \cdot e^{i m \phi}
$$

where $P_{l}^{m}(\cos \theta)$ are Legandres polinomials. Now we rewrite the angular integral in eq. (25) so:

$$
\begin{aligned}
& \int Y_{l_{f} m_{f}}^{*}\left(\mathbf{n}_{r}\right) Y_{1,-\mu^{\prime}}\left(\mathbf{n}_{r}\right) Y_{n, \mu-\mu^{\prime}}^{*}\left(\mathbf{n}_{r}\right) d \Omega= \\
& =\int(-1)^{\frac{m_{f}+\left|m_{f}\right|}{2}}(-1)^{l_{f}} i^{l_{f}} \sqrt{\frac{2 l_{f}+1}{4 \pi} \frac{\left(l_{f}-\left|m_{f}\right|\right) !}{\left(l_{f}+\left|m_{f}\right|\right) !}} P_{l_{f}}^{\left|m_{f}\right|}(\cos \theta) \cdot e^{-i m_{f} \phi} \times \\
& \times \quad(-1) \frac{\frac{-\mu^{\prime}|| \mu^{\prime} \mid}{2}}{i^{1}} \sqrt{\frac{2 \cdot 1+1}{4 \pi} \frac{\left(1-\left|\mu^{\prime}\right|\right) !}{\left(1+\left|\mu^{\prime}\right|\right) !}} P_{1}^{\left|\mu^{\prime \prime}\right|}(\cos \theta) \cdot e^{-i \mu^{\prime} \phi} \times \\
& \times \quad(-1) \frac{\mu-\mu^{\prime}+\left|\mu_{-1}^{\prime}\right|}{2}(-1)^{n} i^{n} \sqrt{\frac{2 n+1}{4 \pi} \frac{\left(n-\left|\mu-\mu^{\prime}\right|\right) !}{\left(n+\left|\mu-\mu^{\prime}\right|\right) !}} P_{n}^{\left|\mu-\mu^{\prime}\right|}(\cos \theta) \cdot e^{i\left(-\mu+\mu^{\prime}\right) \phi} \cdot \sin \theta d \theta d \phi= \\
& =(-1) \frac{m_{f}+\left|m_{f}\right|-\mu^{\prime}+1+\mu-\mu^{\prime}+\left|\mu-\mu^{\prime}\right|}{2}(-1)^{l_{f}+n} i^{l_{f}+n+1} \sqrt{\frac{2 l_{f}+1}{4 \pi} \frac{\left(l_{f}-\left|m_{f}\right|\right) !}{\left(l_{f}+\left|m_{f}\right|\right) !}} \sqrt{\frac{3}{8 \pi}} \sqrt{\frac{2 n+1}{4 \pi} \frac{\left(n-\left|\mu-\mu^{\prime}\right|\right) !}{\left(n+\left|\mu-\mu^{\prime}\right|\right) !}} \times \\
& \times \int_{0}^{2 \pi} e^{i\left(-m_{f}-\mu^{\prime}-\mu+\mu^{\prime}\right) \phi} d \phi \cdot \int_{0}^{\pi} P_{l_{f}}^{\left|m_{f}\right|}(\cos \theta) P_{1}^{1}(\cos \theta) P_{n}^{\left|\mu-\mu^{\prime}\right|}(\cos \theta) \cdot \sin \theta d \theta d \phi .
\end{aligned}
$$

This integral over $\phi$ is non-zero only at fulfillment the following condition:

$m_{f}=-\mu$.

Taking into account $\mu= \pm 1$, we obtain the following restrictions on possible values of $m_{f}$ and $l_{f}$ :

$m_{f}=-\mu= \pm 1, \quad l_{f} \geq 1$,

and also

$n \geq\left|\mu-\mu^{\prime}\right|=\left|m_{f}+\mu^{\prime}\right|$

On such a basis we calculate integral (B2):

$$
\begin{aligned}
& \int Y_{l_{f} m_{f}}^{*}\left(\mathbf{n}_{r}\right) Y_{1,-\mu^{\prime}}\left(\mathbf{n}_{r}\right) Y_{n, \mu-\mu^{\prime}}^{*}\left(\mathbf{n}_{r}\right) d \Omega= \\
& =(-1)^{l_{f}+n-\mu^{\prime}+1+\frac{\left|m_{f}+\mu^{\prime}\right|}{2}} i^{l_{f}+n+1} \sqrt{\frac{3\left(2 l_{f}+1\right)(2 n+1)}{32 \pi} \frac{\left(l_{f}-1\right) !}{\left(l_{f}+1\right) !} \frac{\left(n-\left|m_{f}+\mu^{\prime}\right|\right) !}{\left(n+\left|m_{f}+\mu^{\prime}\right|\right) !}} \times \\
& \times \int_{0}^{\pi} P_{l_{f}}^{1}(\cos \theta) P_{1}^{1}(\cos \theta) P_{n}^{\left|m_{f}+\mu^{\prime \mid}\right|}(\cos \theta) \cdot \sin \theta d \theta d \phi .
\end{aligned}
$$

So, we have just obtained the right part of eq. (25). 


\section{APPENDIX C: COEFFICIENTS $C_{l_{f} l_{h} h^{n}}^{m h^{\prime}}$}

We define the coefficient $C_{l_{f^{\prime} p^{\prime}}^{m}}^{m \mu^{\prime}}$ so:

$$
\begin{aligned}
C_{l_{f} l_{h h^{\prime}}^{m}}^{m \mu^{\prime}} & =(-1)^{l_{f}+n+1-\mu^{\prime}+\frac{\left|m+\mu^{\prime}\right|}{2}}\left(n, 1, l_{p h} \mid-m-\mu^{\prime}, \mu^{\prime},-m\right) \times \\
& \times \sqrt{\frac{\left(2 l_{f}+1\right)(2 n+1)}{32 \pi} \frac{\left(l_{f}-1\right) !}{\left(l_{f}+1\right) !} \frac{\left(n-\left|m+\mu^{\prime}\right|\right) !}{\left(n+\left|m+\mu^{\prime}\right|\right) !}}
\end{aligned}
$$

At $l_{f}=1, l_{p h}=1$ and $n=0$ we have:

$m=-\mu^{\prime}= \pm 1$

and the coefficient $C_{l_{f} \mu^{\prime} h^{h}}^{m \mu^{\prime}}$ is:

$C_{110}^{m \mu^{\prime}}=-\sqrt{\frac{3}{64 \pi}} \cdot\left(011 \mid 0, \mu^{\prime},-m\right)$.

At $l_{f}=1, l_{p h}=1$ and $n=1$, the property (C2) is fulfilled and we obtain:

$C_{111}^{m \mu^{\prime}}=\sqrt{\frac{9}{64 \pi}} \cdot\left(111 \mid 0, \mu^{\prime},-m\right)$

At $l_{f}=1, l_{p h}=1$ and $n=2$, the property (C2) is not fulfilled and

$C_{112}^{m \mu^{\prime}}=(-1)^{-\mu^{\prime}+\frac{\left|m+\mu^{\prime}\right|}{2}} \sqrt{\frac{15}{64 \pi} \frac{\left(2-\left|m+\mu^{\prime}\right|\right) !}{\left(2+\left|m+\mu^{\prime}\right|\right) !}} \cdot\left(211 \mid-m-\mu^{\prime}, \mu^{\prime},-m\right)$.

Rewrite these coefficients at different $m= \pm 1$ and $\mu^{\prime}= \pm 1$ :

$$
\begin{aligned}
C_{112}^{-1-1} & =\frac{1}{16} \sqrt{\frac{5}{2 \pi}} \cdot(211 \mid 2,-1,1), & C_{112}^{-11} & =-\frac{1}{8} \sqrt{\frac{15}{\pi}} \cdot(211 \mid 011), \\
C_{112}^{1-1} & =-\frac{1}{8} \sqrt{\frac{15}{\pi}} \cdot(211 \mid 0,-1,-1), & C_{112}^{11} & =\frac{1}{16} \sqrt{\frac{5}{2 \pi}} \cdot(211 \mid-2,1,-1) .
\end{aligned}
$$

Substituting here values (A1) for the Clebsh-Gordan coefficients, we find:

$$
\begin{array}{llll}
C_{110}^{-1-1}=0, & C_{110}^{-11}=-\frac{1}{8} \sqrt{\frac{3}{2 \pi}}, & C_{110}^{1-1}=-\frac{1}{8} \sqrt{\frac{3}{2 \pi}}, & C_{110}^{11}=0, \\
C_{111}^{-1-1}=0, & C_{111}^{-11}=-\frac{3}{8} \sqrt{\frac{1}{2 \pi}}, & C_{111}^{1-1}=\frac{3}{8} \sqrt{\frac{1}{2 \pi}}, & C_{111}^{11}=0, \\
C_{112}^{-1-1}=\frac{1}{16} \sqrt{\frac{3}{2 \pi}}, & C_{112}^{-11}=-\frac{1}{8} \sqrt{\frac{3}{2 \pi}}, & C_{112}^{1-1}=-\frac{1}{8} \sqrt{\frac{3}{2 \pi}}, & C_{112}^{11}=\frac{1}{16} \sqrt{\frac{3}{2 \pi}} .
\end{array}
$$




\section{APPENDIX D: FUNCTIONS $f_{l_{f} n}^{m \mu^{\prime}}(\theta)$}

Let's consider the function $f_{l_{f} n}^{m \mu^{\prime}}(\theta)$ :

$f_{l_{f} n}^{m \mu^{\prime}}(\theta)=P_{l_{f}}^{1}(\cos \theta) P_{1}^{1}(\cos \theta) P_{n}^{\mid m+\mu^{\prime \mid}}(\cos \theta)$.

At $l_{f}=1$ and $n=0,1,2$ we obtain:

$f_{10}^{m \mu^{\prime}}(\theta)=P_{1}^{1}(\cos \theta) P_{1}^{1}(\cos \theta) P_{0}^{\left|m+\mu^{\prime}\right|}(\cos \theta)$,

$f_{11}^{m \mu^{\prime}}(\theta)=P_{1}^{1}(\cos \theta) P_{1}^{1}(\cos \theta) P_{1}^{\left|m+\mu^{\prime}\right|}(\cos \theta)$,

$f_{12}^{m \mu^{\prime}}(\theta)=P_{1}^{1}(\cos \theta) P_{1}^{1}(\cos \theta) P_{2}^{\mid m+\mu^{\mid}}(\cos \theta)$.

At different $m= \pm 1$ and $\mu^{\prime}= \pm 1$ we find:

$$
\begin{aligned}
& f_{10}^{-1,-1}(\theta)=P_{1}^{1}(\cos \theta) P_{1}^{1}(\cos \theta) P_{0}^{2}(\cos \theta)=0, \\
& f_{10}^{-11}(\theta)=P_{1}^{1}(\cos \theta) P_{1}^{1}(\cos \theta) P_{0}^{0}(\cos \theta)=\sin ^{2} \theta, \\
& f_{10}^{1-1}(\theta)=P_{1}^{1}(\cos \theta) P_{1}^{1}(\cos \theta) P_{0}^{0}(\cos \theta)=\sin ^{2} \theta, \\
& f_{10}^{11}(\theta)=P_{1}^{1}(\cos \theta) P_{1}^{1}(\cos \theta) P_{0}^{2}(\cos \theta)=0, \\
& f_{11}^{-1-1}(\theta)=P_{1}^{1}(\cos \theta) P_{1}^{1}(\cos \theta) P_{1}^{2}(\cos \theta)=0, \\
& f_{11}^{-11}(\theta)=P_{1}^{1}(\cos \theta) P_{1}^{1}(\cos \theta) P_{1}^{0}(\cos \theta)=\sin ^{2} \theta \\
& f_{11}^{1-1}(\theta)=P_{1}^{1}(\cos \theta) P_{1}^{1}(\cos \theta) P_{1}^{0}(\cos \theta)=\sin ^{2} \theta \\
& f_{11}^{11}(\theta)=P_{1}^{1}(\cos \theta) P_{1}^{1}(\cos \theta) P_{1}^{2}(\cos \theta)=0, \\
& f_{12}^{-1-1}(\theta)=P_{1}^{1}(\cos \theta) P_{1}^{1}(\cos \theta) P_{2}^{2}(\cos \theta)=3 \sin ^{4} \theta, \\
& f_{12}^{-11}(\theta)=P_{1}^{1}(\cos \theta) P_{1}^{1}(\cos \theta) P_{2}^{0}(\cos \theta)=\frac{1}{2} \sin ^{2} \theta\left(3 \cos ^{2} \theta-1\right), \\
& f_{12}^{1-1}(\theta)=P_{1}^{1}(\cos \theta) P_{1}^{1}(\cos \theta) P_{2}^{0}(\cos \theta)=\frac{1}{2} \sin ^{2} \theta\left(3 \cos ^{2} \theta-1\right), \\
& f_{12}^{11}(\theta)=P_{1}^{1}(\cos \theta) P_{1}^{1}(\cos \theta) P_{2}^{2}(\cos \theta)=3 \sin ^{4} \theta
\end{aligned}
$$

\section{REFERENCES}

[1] Dyakonov MI, Gornyi IV. Electromagnetic radiation by a tunneling charge. Phys Rev Lett 1996; 76: 3542-5.

[2] Dyakonov MI. Bremsstrahlung spectrum in $\alpha$ decay. Phys Rev C 1999; 60: 037602-4. Available from: nucl-th/9903016

[3] Takigawa $\mathrm{N}$, Nozawa $\mathrm{Y}$, Hagino $\mathrm{K}$, Ono A, Brink DM. Bremsstrahlung in $\alpha$ decay. Phys Rev C 1999; C59: 593-7. Available from: nucl-th/9809001

[4] Boie $\mathrm{H}$, Scheit $\mathrm{H}$, Jentschura UD, et al. Bremsstrahlung in $\alpha$ decay reexamined. Phys Rev Lett 2007; 99: 022505. Available from: arXiv:0706.2109

[5] Jentschura UD, Milstein AI, Terekhov IS, Boie H, Scheit H, Schwalm D. Quasiclassical description of bremsstrahlung accompanying $\alpha$ decay including quadrupole radiation. Phys Rev C 2008; 77: 014611-7.

[6] Bertulani CA, De Paula DT, Zelevinsky VG. Bremsstrahlung radiation by a tunneling particle: a time-dependent description. Phys Rev C 1999; 60: 031602-4. Available from: nucl-ex/9812009

[7] Misicu S, Rizea M, Greiner W. Emission of electromagnetic radiation in $\alpha$-decay. J Phys G 2001; 27: 993-1003.

[8] Dijk W, Nogami Y. Model study of bremsstrahlung in alpha decay. Few-bod Syst Suppl 2003; 14: 229-32.

[9] Serot O, Carjan N, Strottman D. Transient behaviour in quantum tunneling: time-dependent approach to alpha decay. Nucl Phys A 1994; 569: 562-74.

[10] Dijk W, Nogami Y. Novel expression for the wave function of a decaying quantum systems. Phys Rev Lett 1999; 83: 2867-71.

[11] Dijk W, Nogami Y. Analytical approach to the wave function of a decaying quantum system. Phys Rev C 2002; 65: 024608-14.

Ivlev B, Gudkov V. New enhanced tunneling in nuclear processes. Phys Rev C 2004; 69: 037602-4. Available from: nucl-th/0307012 Flambaum VV, Zelevinsky VG. Quantum Münchhausen effect in tunneling. Phys Rev Lett 1999; 83: 3108-11. Available from: nuclth/9812076

Papenbrock T, Bertsch GF. Bremsstrahlung in $\alpha$-decay. Phys Rev Lett 1998; 80: 4141-4. Available from: nucl-th/9801044.

Tkalya EV. Bremsstrahlung spectrum for $\alpha$ decay and quantum tunneling. JETP 1999; 89: 208-18.

Tkalya EV. Bremsstrahlung in $\alpha$-decay and "interference of space regions". Phys Rev C 1999; 60: 054612-5.

So W, Kim Y. Energy and charge dependency for bremsstrahlung in $\alpha$-decay. J Korean Phys Soc 2000; 37: 202-8.

Denisov V Yu, Ikezoe H. Alpha-nucleus potential for alpha-decay and sub-barrier fusion. Phys Rev C 2005; 72: 064613-9. Available from: nucl-th/0510082

Maydanyuk SP, Olkhovsky VS. Does sub-barrier bremsstrahlung in $\alpha$-decay of ${ }^{210}$ Po exist? Prog Theor Phys 2003; 109: 203-11. Available from: nucl-th/0404090

Maydanyuk SP, Belchikov SV. Bremsstrahlung in alpha-decay: angular analysis of spectra. Prob At Sci Technol Ser Nucl Phys Invest 2004; 44: 19-21: Available from: nucl-th/0404013

Maydanyuk SP, Olkhovsky VS. Angular analysis of bremsstrahlung in $\alpha$-decay. Eur Phys J A 2006; 28: 283-94. Available from: nucl-th/0408022.

Giardina G, Fazio G, Mandaglio G, et al. Bremsstrahlung emission accompanying alpha-decay of ${ }^{214}$ Po. Eur Phys J A 2008; 36: 31-6.

Giardina G, Fazio G, Mandaglio G, et al. Bremsstrahlung emission during $\alpha$-decay of ${ }^{226} \mathrm{Ra}$. Mod Phys Lett A 2008; 23: 2651-63. Available from: arXiv:0804.2640 
[24] Buck B, Merchant AC, Perez SM. Half-lives of favored alpha decays from nuclear ground states. At Nucl Dat Tables 1993; 54: 53-74.

[25] Bogoliubov NN, Shirkov DV. Kvantovie polya. Nauka: Mockva 1980 [in Russian].

[26] Landau LD, Lifshitz EM. Kvantovaya M, kurs Teoreticheskoi F. Quantum mechanics, course of theoretical physics. Nauka Mockva, 1989; vol. 3. [in Russian; eng. variant: Pergamon], Oxford: UK 1982.

[27] Eisenberg JM, Greiner W. Mehanizmi vozbuzhdenia yadra. Electromagnitnoie i slaboie vzaimodeistviya (Excitation Mechanisms of Nucleus). Atomizdat: Moskva 1973; vol. 2. [in Russian; Engl.: Excitation mechanisms of the nucleus. Electromagnetic and weak interactions]. North-Holl and publishing company, AmsterdamLondon, 1970.

[28] Berestetsky VB, Lifshitz EM, Pitaevsky LP. Kvantovaya Elektrodinamika, kurs Teoreticheskoi Fiziki. (Quantum electrodynamics, course of Theoretical Physics). Nauka: Mockva 1989; vol. 4. [in Russian; eng Variant]: Pergamon, Oxford, Uk, 1982;: p. 652.

[29] Huizenga JR, Igo G. Theoretical reaction cross sections for alpha particles with an optical model. Nucl Phys 1962; 29: 462-73.

[30] Nolte M, Machner H, Bojowald J. Global optical potential for $\alpha$ particles with energies above $80 \mathrm{MeV}$. Phys Rev C 1987; 36: 13126.

[31] Buck B, Merchant AC, Perez SM. $\alpha$-decay calculations with a realistic potential. Phys Rev C 1992; 45: 2247-53.

[32] Atzrott U, Mohr P, Abele H, Hillenmayer C, Staudt G. Uniform $\alpha$ nucleus potential in a wide range of masses and energies. Phys Rev C 1996; 53: 1336-47.

[33] Blendowske R, Fliessbach T, Walliser H. Nuclear decay modes. Institute of Physics: Bristol 1996.

[34] Khoa D T. $\alpha$-nucleus optical potential in the double-folding model. Phys Phev C 2001; 63: 034007-15.

[35] Moustabchir R, Royer G. Analytic expressions for the proximity energy, the fusion process and the $\alpha$ emission. Nucl Phys A 2001; 683: 266-78.

[36] Demetriou P, Grama C, Goriely S. Improved global $\alpha$-optical model potentials at low energies. Nucl Phys A 2002; 707: 253-76.

[37] Avrigeanu M, Oertzen W, Plompen AJM, Avrigeanu V. Optical model potentials for $\alpha$-particles scattering around the Coulomb barrier on A 100 nuclei. Nucl Phys A 2003; 723: 104-26.

[38] Basu DN. Role of effective interaction in nuclear disintegration processes. Phys Lett B 2003; 566: 90-7.
[39] Belgya T, Bersillon O, Capote R, et al. Handbook for calculations of nuclear reaction data, RIPL-2. IAEA-TECDOC-1506, IAEA: Vienna 2006. Available from: http://www-nds.iaea.org/RIPL-2

[40] $\mathrm{Xu} \mathrm{C}$, Ren Z. Systematical calculation of $\alpha$ decay half-lives by density-dependent cluster model. Nucl Phys A 2005; 753: 174-85.

[41] Zhang HF, Royer G. $\alpha$-particle preformation in heavy nuclei and penetration probability. Phys Rev C 2008; 77: 054318-7.

[42] Bhattacharya M, Gangopadhyay G. $\alpha$-decay lifetime in superheavy nuclei with A > 282. Phys Rev C 2008; 77: 047302-3.

[43] Xu C, Ren Z. New deformed model of $\alpha$-decay half-lives with a microscopic potential. Phys Rev C 2006; 73: 041301(R)-5.

[44] Mohr P. $\alpha$-nucleus potentials, $\alpha$-decay half-lives, and shell closures for superheavy nuclei. Phys Rev C 2006; 73: 031301(R)-5.

[45] Mohr P. $\alpha$-nucleus potentials for the neutron-deficient $p$ nuclei. Phys Rev C 2000; 61: 045802-5.

[46] Hoyler F, Mohr P, Staudt G. Alpha-cluster states of ${ }^{212}$ Po in a realistic potential model. Phys Rev C 1994; 50: 2631-4.

[47] Varga K, Lovas RG, Liotta RJ. Absolute alpha decay width of ${ }^{212} \mathrm{Po}$ in a combined shell and cluster model. Phys Rev Lett 1992; 69: 37 40.

[48] Mohr P. $\alpha$-cluster states in intermediate mass nuclei. Open Nucl Part Phys J 2008; 1: 1-8. Available from: http://www.bentham.org/ open/tonppj/openaccess 2 .htm

[49] D'Arrigo A, Eremin NV, Fazio J, et al. Investigation of bremsstrahlung emission in $\alpha$-decay of heavy nuclei. Phys Lett B 1994; 332: 25-30.

[50] Kasagi J, Yamazaki H, Kasajima N, Ohtsuki T, Yuki H. Bremsstrahlung emission in $\alpha$-decay and tunneling motion of $\alpha$ particle. J Phys G 1997; 23: 1451-7.

[51] Kasagi J, Yamazaki H, Kasajima N, Ohtsuki T, Yuki H. Bremsstrahlung in $\alpha$-decay of ${ }^{210}$ Po: do $\alpha$-particles emit photons in tunneling? Phys Rev Lett 1997; 79: 371-4.

[52] Eremin NV, Fazio G, Giardina G. Comment on "Bremsstrahlung in $\alpha$-decay of ${ }^{210} \mathrm{Po}$ : do $\alpha$-particles emit photons in tunneling?" Phys Rev Lett 2000; 85: 3061 .

[53] Kasagi J, Yamazaki H, Kasajima N, Ohtsuki T, Yuki H. Replay on Comment on "Bremsstrahlung in $\alpha$-decay of ${ }^{210} \mathrm{Po}$ : do $\alpha$-particles emit photons in tunneling?" Phys Rev Lett 2000; 85: 3062.

[54] Ohtsuki T, Yuki H, Hirose K, Mitsugashira T. Status of the electron accelerator for radioanalytical studies at Tohoku University. Czech J Phys 2006; 56: D391-8. 